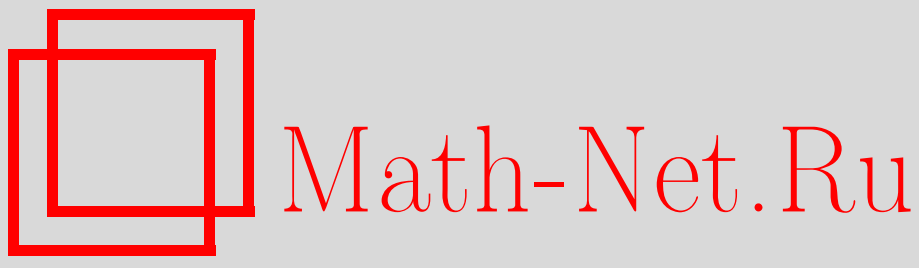

М. Л. Гервер, Е. А. Кудрявцева, Универсальная последовательность в классической задаче обращения годографа, Матем. сб., 1997, том 188, номер 4, 3-56

DOI: https://doi.org/10.4213/sm215

Использование Общероссийского математического портала Math-Net.Ru подразумевает, что вы прочитали и согласны с пользовательским соглашением http://www . mathnet.ru/rus/agreement

Параметры загрузки:

IP: 3.81 .55 .215

26 апреля 2023 г., 13:59:22 
УДК $517+550.34$

\author{
М. Л. Гервер, Е. А. Кудрявцева
}

\title{
Универсальная последовательность в классической задаче обращения годографа
}

\begin{abstract}
Обращение годограффа - фундаментальная задача математической геофизики: на (и вблизи) поверхности Земли происходят взрьвы и землетрясения, приборы регистрируют сигналы от них, и по временам пробега сигналов от источников до приемников (по годографу) нужно найти скорость упругих волн внутри Земли. После пионерских работ начала века и детальных исследований 60 -х годов трудно было ожидать появления принципиально новых результатов в этой задаче в ее классической постановке, когда скорость волн предполагается зависящей только от глубины. Оказалось однако, что считать эту постановку исчерпанной преждевременно. Доказанные в статье теоремы об универсальной последовательности и экстремальных свойствах дискретных мер, вероятно, удивят специалистов по обратным задачам и заинтересуют знатоков и любителей экстремалњных задач.

Библиография: 20 названий.
\end{abstract}

\section{§1. Введение}

Недавно были установлены неожиданные связи между задачей обрашения годографа и, на первый взгляд, далекими от нее областями: геометрией устойчивых многочленов, рациональными аппроксимациями, чебышёвскими системами и вполне положительньми ядрами. Обнаружение и изучение этих связей и, особенно, работы [1]-[3] привели к сушественному продвижению в задаче обрашения годографа [4], [5]. В статье после обзора установленных ранее фактов доказаны теоремы, сформулированные в заметках [4], [5] и докладах [6], [7], а также ряд новых теорем.

\section{§2. Какого типа задачи решаются в статье?}

Прежде чем приступить к систематическому изложению результатов (оно начнется в $\S 3$ ), расскажем, какого типа задачи решаются в статье.

Начнем с простого примера. Возьмем три табличных интеграла:

$$
\int_{0}^{1} d s=1, \quad \int_{0}^{1} s d s=\frac{1}{2}, \quad \int_{0}^{1} s^{2} d s=\frac{1}{3} .
$$

Рассмотрим стилтьесовские меры $d a(s) \geqslant 0$ на $[0,1]$, для которых

$$
I_{0}=\int_{0}^{1} d a(s)=1, \quad I_{1}=\int_{0}^{1} s d a(s)=\frac{1}{2}
$$

Работа выполнена при финансовой поддержке Российского фонда фундаментальных исследований (грант № 96-01-01852). 
и найдем максимум и минимум интеграла $I_{2}=\int_{0}^{1} s^{2} d a(s)$ по всем таким мерам. Нетрудно проверить ${ }^{\langle 1\rangle}$ (здесь и далее ссылка вида $\langle k\rangle$ отсылает к комментарию с

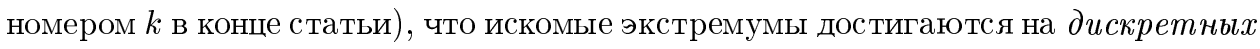
мерах

$$
\begin{gathered}
d a_{1}(s)=\frac{\delta(s)+\delta(s-1)}{2} d s, \quad d a_{2}(s)=\delta\left(s-\frac{1}{2}\right) d s \\
\max I_{2}=\int_{0}^{1} s^{2} d a_{1}(s)=\frac{1}{2}, \quad \min I_{2}=\int_{0}^{1} s^{2} d a_{2}(s)=\frac{1}{4}
\end{gathered}
$$

Этот несложный результат (легко обобщаемьй с помощью теории канонических представлений Маркова-Крейна [8]) сродни основньм теоремам нашей статьи. Теоремы сформулированы в $\S 5$ и доказаны в $\S \S 6-10$. Необходимые для их понимания геофизические сведения собраны в подготовительных $\S 3$ и $§ 4$.

Математическая ситуация такова. Некоторое правило

$$
d a \mapsto g=g(d a, t), \quad h=h(d a, t), \quad t \in \Delta
$$

(точнее оно описано чуть ниже), сопоставляет каждой неотрицательной мере Стилтьеса

$$
d a=d a(s), \quad s>0, \quad \int_{0}^{\infty} d a(s)=1,
$$

две функции $(g$ и $h)$ на отрезке $\Delta$. Меры $d a$ пропускаются через своеобразный фильтр, а именно: фиксируется некоторая положительная функция (мажсоранта) $M(t), t \in \Delta$, и $d a$ считается прошедшей через фильтр, если

$$
g(d a, t) \leqslant M(t) \text { при всех } t \in \Delta .
$$

На множестве $(* *)$ мер $d a$ рассматривается однопараметрическое (зависящее от параметра $t$ ) семейство экстремальных задач:

$$
\text { найтu } \max h(d a, t), \quad t \in \Delta .
$$

Правило $(*)$ состоит в следуюшем. Рассматривается некоторый класс $\mathbb{K}$ функций (ядер) $K(s, t), s>0,-\infty<\alpha \leqslant t \leqslant \beta \leqslant \infty$, со свойствами, аналогичными свойствам ядра Коши

$$
C(s, t)=\frac{1}{s+t}, \quad s>0, \quad t \geqslant 0
$$

Фиксируются ядро $K(s, t) \in \mathbb{K}$, число $\gamma \in(\alpha, \beta)$ и такая неотрицательная мера Стилтьеса $d \theta$ на $[\alpha, \beta)$, что следующие интегралы сходятся:

$$
\kappa(s, t)=\int_{t}^{\beta} K(s, x) d \theta(x)<\infty, \quad s>0, \quad t \in \Delta=[\alpha, \gamma]
$$


Функции $g$ и $h$ в $(*)$ определяются следующим образом:

$$
g(d a, t)=\int_{0}^{\infty} K(s, t) d a(s), \quad h(d a, t)=\int_{0}^{\infty} \kappa(s, t) d a(s)=\int_{t}^{\beta} g(d a, x) d \theta(x) .
$$

Оказывается тогда, как и в рассмотренном в начале $\S 2$ примере, искомые максимумы $(* * *)$ достигаются на дискретных мерах. Подробнее: при некоторых условиях на $K, d \theta$ и $M(t)$ для каждого $t \in(\alpha, \gamma]$ существуют и однозначно определяются $n=n(t)$ и такой набор чисел $\left\{a_{1}, \ldots, a_{n}, s_{1}, \ldots, s_{n}\right\}$,

$$
a_{j}=a_{j}(t)>0, \quad \sum a_{j}=1, \quad s_{1}=s_{1}(t)>\cdots>s_{n}=s_{n}(t)>0
$$

что максимум $h(d a, t)$ достигается на мере

$$
d a=d a(s)=\sum a_{j} \delta\left(s-s_{j}\right) d s, \quad 1 \leqslant j \leqslant n=n(t) .
$$

Более того, сушествует такая универсальная (определяемая ядром $K$ и мерой $d \theta$ и не зависящая от выбора мажсоранты $M(t))$ невозрастаюшая и сходяшаяся к $\alpha$ последовательность $t_{k} \in[\alpha, \gamma], k \geqslant 1$, что для функции $n(t)$ выполняется неравенство

$$
n(t) \leqslant k \text { при } t \in\left[t_{k}, \gamma\right] .
$$

Интересный, не решенньй пока полностью вопрос: монотонна ли $n(t)$ ? Положительньй ответ на него дан в $\S 11$ при дополнительных ограничениях.

В геофизических приложениях важны ядро Коши $C(s, x)=1 /(s+x)$ и мера $d \theta(x)=(2 / \pi) d \sqrt{x}, 0<x<\infty ;$ для них (при $\alpha=0, \gamma=1$ и $\beta=\infty)$

$$
\kappa(s, t)=\frac{2}{\pi} \int_{t}^{\infty} \frac{d \sqrt{x}}{s+x}=\frac{2}{\pi} \frac{\operatorname{arctg} \sqrt{s / t}}{\sqrt{s}}, \quad t \in(0,1] ; \quad \kappa(s, 0)=\frac{1}{\sqrt{s}} .
$$

В этом случае, которому и посвящена настоящая статья и который, оказывается, тесно связан с полиномами Лежандра

$$
L_{0}(t)=1, \quad L_{1}(t)=t, \quad L_{n}(t)=\left(2-\frac{1}{n}\right) t L_{n-1}(t)-\left(1-\frac{1}{n}\right) L_{n-2}(t)
$$

и квадратурами Гаусса ${ }^{\langle 2\rangle}$, универсальная последовательность $\left\{t_{k}\right\}$ в задаче о

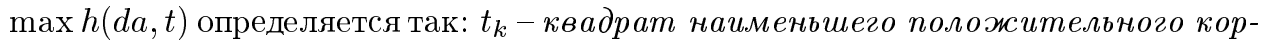
ня полинома Лежандра $L_{2 k+1}(t), k \geqslant 1$.

Этот результат позволяет построить универсальную последовательность $U=\left\{U_{k}\right\}$ в задаче обрашения годограффа более простым способом, чем в [4], [5], положив $U_{k}=1 / \sqrt{1-t_{k}}$ (см. §5).

\section{§3. Классическая задача обращения годографа. Ключевые слова}

Перейдем к систематическому изложению результатов. Начнем с краткой исторической справки и объяснения ключевых слов годограф и волновод (хорошо знакомых геофизикам, но, возможно, неизвестных математикам). 
В начале века задача обрашения годографа была решена Герглотцем [9] и Бейтменом [10] в предположении, что Земля - шар (радиуса $R$ ), скорость волн зависит только от глубинь: $v=v(r), r \in[0, R]$, причем ${ }^{\langle 3\rangle}$

$$
\frac{v(r)}{r} \text { не возрастает на }[0, R] \text { и } \frac{v(r)}{r} \rightarrow \infty \text { при } r \rightarrow 0 .
$$

Задача была сделана в лучевом приближении. При $v=v(r)$ распространяющиеся по принципу Ферма сейсмические лучи (геодезические) - плоские кривые, так что задачу можно рассматривать не в шаре, а в круге. Заменим круг римановой поверхностью $Z$, склеенной из счетного множества кругов с разрезом вдоль радиуса: совершив оборот вокруг центра круга, луч переходит на следуюший лист. Конформное отображение $w=\ln (R / z)$ поверхности $Z$ на полуплоскость упрошает задачу ${ }^{\langle 4\rangle}$. Теперь лучи распространяются в полуплоскости $x, y(y \geqslant 0)$ со скоростью $u(y)$, равной $v(r) / r$, и условие (1) означает, что

$$
u(y) \text { не убъвает при } y \geqslant 0 \text { и } u(y) \rightarrow \infty \text { при } y \rightarrow \infty .
$$

Сформулируем точно задачу обращения годографа в полуплоскости и приведем сводку полученных ранее результатов.

Нормировка. Выберем масштаб на оси $u$ так, чтобы

$$
u(0)=1 .
$$

Годограф. Из точки 0 плоскости $x, y$ в (нижнюю) полуплоскость $y \geqslant 0$ выходят сейсмические лучи, по которым импульс, возникший в момент $t=0$, распространяется со скоростью $u(y)$, завислшей только от $y$. Пусть $L-$ луч, образуюший в точке 0 угол $\alpha$ с осью $y, 0<\alpha<\pi / 2$ (рис. 1 ). Число $p=\sin \alpha$ называется лучевъцм параметром. Пусть $X(p), Y(p)$ - координаты самой глубокой точки луча $L$, а $T(p)$ - время распространения импульса до нее вдоль $L$. Пусть $X(p)$ конечно (это верно для почти всех $p \in(0,1)$ ), тогда луч $L$ состоит из двух дуг (нисходящей и восходящей), симметричных относительно прямой $x=X(p)$, луч $L$ возвращается на прямую $y=0$ в точке $x=2 X(p)$, и время движения импульса по $L$ равно $2 T(p)$.

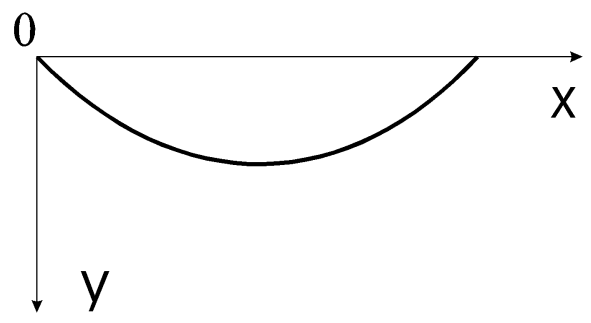

Рис. 1 
ОПРЕДЕЛЕНИЕ. Кривую

$$
x=2 X(p), \quad t=2 T(p), \quad p \in(0,1),
$$

на плоскости $x, t$ обозначим $\Gamma$ и назовем годографом.

Годограф Г считается известным, а и(у) нужно найти.

Формула Бендорфа. Уравнение Абеля. По закону Снелла в любой своей точке $(x, y)$ нисходяшая дуга $L$ образует с осью $y$ угол $\alpha(y)$ такой, что

$$
\sin \alpha(y)=p u(y) .
$$

Вследствие $(5)$ в самой нижней точке $(X(p), Y(p))$ луча $L$, где $\sin \alpha(y)=1$, вьполняется равенство $u(Y(p))=1 / p$, т.е. $u(y)$ и $Y(1 / u)$ - взаимно обратные функции (участкам постоянства одной соответствуют разрывы другой).

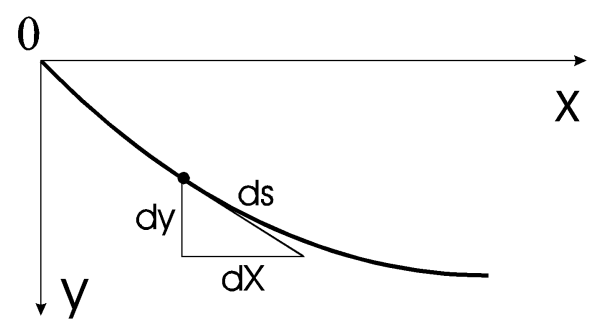

Рис. 2

Поскольку (рис. 2$)$ в точке $(x, y)$ нисходящей дуги луча $L$

$$
\frac{d x}{d y}=\operatorname{tg} \alpha(y), \quad \frac{d y}{d s}=\cos \alpha(y), \quad \frac{d y}{d t}=u(y) \cos \alpha(y),
$$

то $X(p)$ и $T(p)$ равны интегралам от 0 до $Y(p)$ от функций

соответственно, т.е. ввиду (5)

$$
\operatorname{tg} \alpha(y), \quad \frac{1}{u(y) \cos \alpha(y)},
$$

$$
X(p)=\int_{0}^{Y(p)} \frac{p u(y) d y}{\sqrt{1-p^{2} u^{2}(y)}}, \quad T(p)=\int_{0}^{Y(p)} \frac{d y}{u(y) \sqrt{1-p^{2} u^{2}(y)}} .
$$

Так как

$$
d T(p)=p d X(p)
$$

(формула Бендорфа), то задающие кривую (4) функции $X(p)$ и $T(p)$, как правило, определяются по годографу ${ }^{\langle 5\rangle}$, и обращение годографа сводится тогда к решению уравнений (6).

Первое из них - это интегральное уравнение Абеля

$$
X(p)=\int_{0}^{Y(p)} \frac{p u(y) d y}{\sqrt{1-p^{2} u^{2}(y)}}, \quad p \in(0,1) .
$$

При выполнении условия (2) оно однозначно разрешимо:

$$
Y(p)=\frac{2}{\pi} \int_{p}^{1} \frac{X(q) d q}{\sqrt{q^{2}-p^{2}}}, \quad p \in(0,1) .
$$

Это и есть результат Герглотца-Бейтмена: $Y(p)$, а значит, и обратная к $Y(1 / u)$ функция $u(y)$ однозначно определяются по $X(p)$ с помошью явной формулы (8). 
Волноводы. Сушественна ли монотонность $u(y)$ для однозначной разрешимости задачи обращения годографа? Пусть, например, график $u(y)$ имеет такой вид, как на рис. 3. Можно ли определить тогда $u(y)$ по годографу?

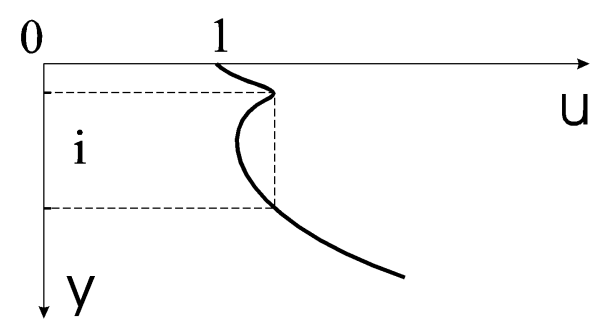

Рис. 3

Интервал $i$ оси $y$ на рис. 3 (так же, как и полоса $y \in i$ на плоскости $x, y$ ) называется волноводом ${ }^{\langle 6\rangle}$. Если заменить $u(y)$ функцией, равной $u(y)$ вне $i$ и равноизмеримой с ней ${ }^{\langle 7\rangle}$ внутри $i$, то лучи вне волновода $i$ и время движения импульса по каждому из них, а значит, и годограф̆ не изменятся [11] ${ }^{\langle 8\rangle}$.

До середины 60-х годов среди геофизиков было распространено убеждение: неоднозначность в определении скорости и(у) по годографу этим и исчерпьвается. Оказалось, однако, что это не так [12], [13]. В [14] подробно исследована неединственность в задаче обращения годографа при наличии $n$ волноводов. Кратко опишем результаты этого исследования для $n=1$.

\section{§4. Неоднозначность в определении скорости по годографу}

Относительно любой из рассматриваемых скоростей $u(y), y \geqslant 0$, предположим следуюшее.

Предположения об $u(y)$. Будем считать $u(y)$ кусочно дваждъ гладжой на каждом конечном отрезке полуоси $y \geqslant 0$ (иньми словами, сама $u(y)$ имеет конечное число разрывов 1-го рода на каждом таком отрезке, и первые две производные $u(y)$ могут не сушествовать или быть разрьвньми в конечном числе точек такого отрезка); в точках разрыва $u(y)=\max \{u(y-0), u(y+0)\}$. Каждая скорость $u(y)$ имеет один волновод $(0, Y)$; по определению это означает (рис. 4$)$, что

$$
\begin{gathered}
u(y) \leqslant u(0)=1, \quad u(y) \not \equiv 1 \text { при } 0<y<Y, \\
u(y)>1 \text { при } y>Y, \quad u(y) \text { не убывает при } y \geqslant Y, \\
u(y) \rightarrow \infty \text { при } y \rightarrow \infty .
\end{gathered}
$$

ЗАмечАниЕ. Тем самым предполагается, что волновод начинается сразу у поверхности (при $y=0$ ); случай, когда он начинается глубже (при $y>0$ ), легко сводится к рассматриваемому. Ширина волновода $Y$ может быть разной для разных $u(y)$, в то время, как мера $m=\operatorname{mes}\{y \in(0, Y), u(y)=1\}-$ одна и та же для всех $u(y)$ с одним и тем же годографом Г. Эта мера равна пределу $\Phi(1-0)$ при $p \rightarrow 1$ определяемой ниже (см. (12)) функции $\Phi(p), p \in(0,1)$. 


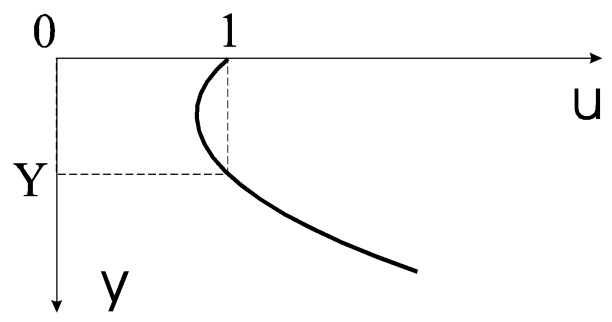

Рис. 4

Преобразование Лежандра годографа Г. Вместо пары функций $X(p)$ и $T(p)$ (см. (4), (6)) удобно рассматривать функцию

$$
\tau(p)=\int_{0}^{Y(p)} \sqrt{u^{-2}(y)-p^{2}} d y, \quad p \in(0,1) .
$$

Это - непрерьвная в интервале $(0,1)$ убываюшая функция; ввиду $(6),(7)$ она связана с $X(p)$ и $T(p)$ следуюшими формулами ${ }^{\langle 9\rangle}$ :

$$
\tau(p)=T(p)-p X(p), \quad \tau^{\prime}(p)=-X(p) .
$$

Согласно (7) и (10), если в точке $(x=2 X(p), t=2 T(p)) \in \Gamma$ кривая $Г$ имеет касательную $k(p)$, то, во-первых, $k(p)$ образует с осью $x$ угол, тангенс которого равен $p$, и, во-вторых, $k(p)$ пересекает ось $t$ в точке $2 \tau(p)$. Таким образом, $2 \tau(p)$ является преобразованием Лежандра годографа Г. Функцию $\tau(p)$ будем считать известной (ср. с $\left.{ }^{\langle 5\rangle}\right)$.

Допустимые функции. Полоса $G$. Масштаб на оси $y$ выберем так (ср. с (3)), чтобы выполнялось нормировочное условие

$$
\tau(1-0)=\int_{0}^{Y} \sqrt{u^{-2}(y)-1} d y=1 .
$$

Функции $u(y)$, удовлетворяющие перечисленньм в начале $\S 4$ предположениям и условию (11), будем назьвать допустимылми.

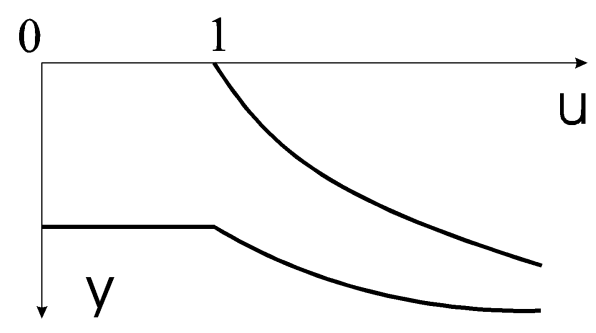

Рис. 5 
Система уравнений (6) эквивалентна интегральному уравнению (9), так что задача обращения годографа сводится в основном к нахождению всех допустимых функций $u(y)$, удовлетворяющих этому уравнению. Согласно [12]-[14] граффики всех таких функций заметают полосу $G$ на плоскости $y, u$ (рис. 5).

Формула-фильтр. Расстояние от оси $u$ до верхней границы полосы $G$ вдоль прямой $u=1 / p, p \in(0,1)$, обозначим $\Phi(p)$. Согласно [12]-[14] $\Phi(p)$ однозначно определяется по $\tau(p)^{\langle 10\rangle}$ :

$$
\Phi(p)=-\frac{2}{\pi} \int_{p}^{1} \frac{d \tau(q)}{\sqrt{q^{2}-p^{2}}}, \quad p \in(0,1),
$$

и для любого допустимого решения $u(y)$ уравнения (9)

$$
Y(p)=\Phi(p)+\Psi(p)
$$

где $\Psi(p)$ выражается через $u(y)$ в волноводе $(0, Y)$

$$
\Psi(p)=\frac{2}{\pi} \int_{0}^{Y} \operatorname{arctg} \sqrt{\frac{u^{-2}(y)-1}{1-p^{2}}} d y, \quad p \in(0,1) .
$$

В формуле $Y(p)=\Phi(p)+\Psi(p)$ заключен следующий способ построения всех допустимых решений уравнения (9).

Возьмем произвольное $Y>0$ и зададим любую допустимую функцию $u(y)$ в волноводе $0<y<Y$. Применив к ней (13), (14), определим $Y(p)$. Если $Y(p)$ не возрастает, получаем $u(y)$ вне волновода (при $y \geqslant Y$ ) как функцию, обратную к $Y(1 / u)$; согласно [12]-[14] полученная $u(y), y \geqslant 0$, удовлетворяет уравнению $(9)$. Если $Y(p)$ не является невозрастающей функцией, то $u(y)$ нельзя продолжить на полуось $y \geqslant Y$ до решения уравнения (9).

Таким образом, формула $Y(p)=\Phi(p)+\Psi(p)$ действует как фильтр: некоторые допустимые $u(y)$ однозначно продолжаются из волновода $0<y<Y$ на полуось (множество таких функиий $и(y)$ обозначим $\mathscr{U})$, другие $u(y)$ не допускают такого продолжения.

\section{§5. Новое в классической задаче обращения годографа}

Универсальная последовательность $U$. Через фильтр (13), (14) можно, в частности, пропускать ступенчатые функции $u(y), 0<y<Y$, удовлетворяющие условиям (3), (11) и принимаюшие значения $u \in(0,1]$, причем (по замечанию в начале $\S 4) \operatorname{mes}\{y \in(0, Y), u(y)=1\}=\Phi(1-0)$. Отнесем такую функцию к $\mathscr{U}_{k}$, если она принадлежит множеству $\mathscr{U}$ (определенному в конще $\S 4$ ) и принимает в интервале $(0, Y)$ не более $k$ значений, не равных 1.

Сформулируем несколько теорем, раскрывающих роль функций из $\mathscr{U}_{k}$ в описании нижней границы полосы $G$ (рис. 5$)^{\langle 11\rangle}$. Их доказательства будут даны в следуюших параграфах.

Первые две теоремы содержат определение и алгоритм построения универсальной последовательности $U$.

Обозначим через $H(p)$ расстояние между верхней и нижней границами полосы $G$ вдоль прямой $u=1 / p, p \in(0,1)$. По определению множества $\mathscr{U}$ (ср. с (15) в теореме 1, см. ниже)

$$
H(p)=\sup \Psi(p) \text { по всем } u(y) \in \mathscr{U} .
$$


Теорема 1. Существует последовательность $U=\left\{U_{k}\right\}$,

$$
U_{0}=+\infty>U_{1}>U_{2}>\cdots>U_{k}>\cdots, \quad U_{k} \rightarrow 1
$$

обладающая следующим свойством: при $u=1 / p \in\left[U_{k}, U_{k-1}\right), k \geqslant 1$,

$$
H(p)=\sup \Psi(p) \quad \text { no } \quad u(y) \in \mathscr{U}_{k} .
$$

Теорема 2. Последовательность $U$ универсальна, т.е. не зависит от $\tau(p)$,

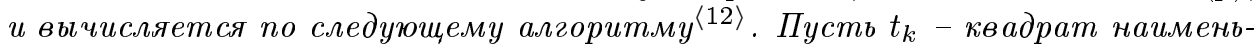
шего положительного корня полинома Лежандра $L_{2 k+1}$. Тогда

$$
U_{k}=\frac{1}{\sqrt{1-t_{k}}}
$$

Ввиду (16) числа $U_{k}$ больше 1 и убъцвают (это следует из чередования положительных корней полиномов Лежандра $L_{2 k-1}$ и $\left.L_{2 k+1}\right)$. Легко доказать ${ }^{\langle 13\rangle}$, что $U_{k} \rightarrow 1$ при $k \rightarrow \infty$, причем

$$
U_{k}-1=O\left(\frac{1}{k^{2}}\right)
$$

Чтобы представить себе, насколько бълтро убъвают разности $U_{k}-1$, умножим их на $10^{4}$ и с помошью таблиц А. С. Кронрода [15] вычислим (по алгоритму теоремы 2$)$ ближайшие к $\left(U_{k}-1\right) 10^{4}$ целые числа, $1 \leqslant k \leqslant 19$ :

$$
5811,1867,942,571,384,277,209,163,131,108
$$$$
90,76,66,57,50,44,39,35,32 \text {. }
$$

В соответствии с (18) числа $U_{k}$ выписываются в дальнейшем с точностью до $5 \cdot 10^{-5}$

$$
1.5811,1.1867,1.0942,1.0571,1.0384,1.0277 \text { и т. д. }
$$

Однопараметрическое семейство $u_{P}(y)$. В следуюших теоремах вводится семейство функций $u_{P}(y)$, для которых нижняя граница полосы $G$ является огибающей.

ТЕорема 3. Для любой точки $P=(y, u), u>1$, нижней границы полосы $G$ существует скоростная функция $u_{P}(y)$ с годографом $\Gamma$, график которой содержит $P$.

ТЕОРема 4. Вне волновода функиия и $($ (у) определена однозначно, а в волноводе - с точностью до равноизмеримости.

ТеОРема 5. Если кордината и точки $P=(y, u)$ принадлежит $\left[U_{k}, U_{k-1}\right)$, то в волноводе $u_{P}(y) \in \mathscr{U}_{k}$.

ОБОзНАчЕнИЕ. При нормировке (3) значения функции $u_{P}(y)$ в волноводе не превосходят 1 ; число ее значений, меньиих 1 , обозначим через $n_{P}$.

Теорему 5 можно переформулировать следуюшим образом. 
TЕОРема $5^{\prime}$. Справедливо неравенство $n_{P} \leqslant k$, где $k=k(u)$ убъвает при возрастании координаты и точки $P=(y, u)$ :

$$
\begin{aligned}
& k=10 \text { при } 1.0108 \leqslant u<1.0131, \quad k=9 \text { при } 1.0131 \leqslant u<1.0163, \\
& k=8 \quad \text { при } 1.0163 \leqslant u<1.0209, \quad k=7 \text { при } 1.0209 \leqslant u<1.0277 \text {, } \\
& k=6 \quad \text { при } 1.0277 \leqslant u<1.0384, \quad k=5 \text { при } 1.0384 \leqslant u<1.0571 \text {, } \\
& k=4 \quad \text { при } 1.0571 \leqslant u<1.0942, \quad k=3 \text { при } 1.0942 \leqslant u<1.1867 \text {, } \\
& k=2 \quad \text { при } 1.1867 \leqslant u<1.5811, \quad k=1 \text { при } 1.5811 \leqslant u<+\infty .
\end{aligned}
$$

Пояснение. Функция $u_{P}(y)$ в теоремах $3-5$ определена в волноводе $c$ точноствю до равноизмеримости: скоростные функции с одним и тем же годографом, равноизмеримые в волноводе $(0, Y)$, совпадают при $y>Y$. Поэтому можно считать, что модель Земли, соответствуюшая $u_{P}(y)$, имеет в волноводе ровно $n_{P}$ слоев, в которых скорость постоянна и - при нормировке (3) - меньше 1. Согласно (19), если пренебречь участком $1 \leqslant u<1.0108$, то для описания нижней границы полосы $G$ достаточно рассматривать модели, имеющие в волноводе не более десяти таких слоев. При этом для $u \geqslant 1.5811$ нижняя граница полосы $G$ совпадает с графиком скоростной функции $u_{P}(y)$, принимающей в волноводе лишь одно значение, меньшее 1, и имеющей среди всех таких функций самый широкий волновод; участком $1.1867 \leqslant u<1.5811$ нижней границы $G$ "заведуют" модели с $n_{P} \leqslant 2$ (т.е. модели с волноводами, содержащими не более двух слоев, в которых скорость меньше 1); участком $1.0942 \leqslant u<1.1867$ - модели с $n_{P} \leqslant 3$ и т. д.

Преобразование функций $Y(p), \Phi(p)$ и $\Psi(p)$. Мера $d a$. Цель последующих преобразований - дать определение мажоранты $M(t)$ и, получив критерий принадлежности допустимой скорости $u(y)$ к множеству $\mathscr{U}$ (введенному в конще $\S 4$ ), переформулировать теоремы $1-5$ в терминах $\S 2$. Положим

$$
q=\sqrt{1-p^{2}}, \quad p \in[0,1],
$$

заменим $Y(p), \Phi(p)$ и $\Psi(p)$ в (12)-(14) на $y(q), \phi(q)$ и $\psi(q)$ и перепишем (13) в виде

$$
y(q)=\phi(q)+\psi(q), \quad q \in(0,1) .
$$

Кроме того, положим $\sqrt{u^{-2}(y)-1}=f(y), y \in(0, Y)$, тогда

$$
\psi(q)=\frac{2}{\pi} \int_{0}^{Y} \operatorname{arctg} \frac{f(y)}{q} d y, \quad q \in(0,1)
$$

Функцию, столшую под знаком интеграла в (21), продифференцируем по $q$

$$
\left(\operatorname{arctg} \frac{f(y)}{q}\right)^{\prime}=\frac{-f(y)}{f^{2}(y)+q^{2}}
$$

Для $f^{2}(y)$ и $q^{2}$ введем специальные обозначения:

$$
s(y)=f^{2}(y)=u^{-2}(y)-1, \quad y \in(0, Y) ; \quad t=q^{2}=1-p^{2} .
$$


ЗАмечание. В дальнейшем $t$ и $x$ уже не будут обозначать время и әпицентральное расстояние, а будут использоваться как в 2 .

Согласно (21), (22) и (23)

$$
\begin{gathered}
\psi(q)=\frac{2}{\pi} \int_{0}^{Y} \operatorname{arctg} \frac{f(y)}{q} d y=\frac{2}{\pi} \int_{0}^{Y} \operatorname{arctg} \sqrt{\frac{s(y)}{t}} d y \\
-\frac{\pi}{2} \psi^{\prime}(q)=\int_{0}^{Y} \frac{f(y) d y}{f^{2}(y)+q^{2}}=\int_{0}^{Y} \frac{\sqrt{s(y)} d y}{s(y)+t} .
\end{gathered}
$$

При этом ввиду нормировочного условия (11)

$$
\int_{0}^{Y} f(y) d y=\int_{0}^{Y} \sqrt{s(y)} d y=1
$$

Положим $\mu(s)=\operatorname{mes}\{y \in(0, Y) \mid s(y) \leqslant s \Leftrightarrow u(y) \geqslant 1 / \sqrt{s+1}\}$,

$$
d a(s)=\sqrt{s} d \mu(s)
$$

т.е. будем понимать под $a(s), s>0$, неубывающую функцию, равную интегралу от $f(y)$ по множеству тех $y \in(0, Y)$, где $s(y) \leqslant s$, введем функцию

$$
\kappa(s, t)=\frac{2}{\pi} \frac{\operatorname{arctg} \sqrt{s / t}}{\sqrt{s}}, t>0 ; \quad \kappa(s, 0)=\frac{1}{\sqrt{s}}
$$

и перепишем $(24),(25)$ и $(26)$ в виде

$$
\psi(\sqrt{t})=\int_{0}^{\infty} \kappa(s, t) d a(s), \quad-\frac{\pi}{2} \psi^{\prime}(\sqrt{t})=\int_{0}^{\infty} \frac{d a(s)}{s+t}, \quad \int_{0}^{\infty} d a(s)=1 .
$$

Мажоранта. Положим

$$
m(q)=\phi^{\prime}(q)
$$

в точках $q$ множества $\mathscr{C}$ непрерывности производной $\phi^{\prime}(q)$ и

$$
m(q)=\underline{\lim } \phi^{\prime}(s) \text { при } s \rightarrow q, \quad s \in \mathscr{C},
$$

в остальных $q \in[0,1]$ (согласно $\S 4$ их конечное число вне любой окрестности точки $q=1)$. Ввиду $(30)$ и $(31)$ функция $m(q)$ полунепрерьвна снизу в каждой точке $q \in[0,1]$. Согласно (20)

$$
y^{\prime}(q)=\phi^{\prime}(q)+\psi^{\prime}(q), \quad q \in \mathscr{C} \subseteq[0,1],
$$

а так как $y(q)$ - неубъвающая функция, то

$$
-\psi^{\prime}(q) \leqslant m(q), \quad q \in[0,1] .
$$


ОПРЕДЕЛЕНИЕ. Назовем мажсорантой функцию

$$
M(t)=\frac{\pi}{2} m(\sqrt{t}), \quad t \in[0,1] .
$$

Мажоранту $M(t)$ считаем известной, поскольку она однозначно определяется по $\Phi(p)$. Ввиду (29), (32) и (33) получаем

Критерий принадлежности к множеству $\mathscr{U}$. Допустимая функция $u(y)$, $y \in(0, Y)$, тогда и только тогда принадлежит множеству $\mathcal{U}$ (см. конец $\S 4$ ), когда соответствующая ей мера $d a(s)$ из (27) удовлетворяет неравенству

$$
\int_{0}^{\infty} \frac{d a(s)}{s+t} \leqslant M(t), \quad t \in[0,1] .
$$

Левую часть (34) обозначим через $g(d a, t)$ :

$$
g(d a, t)=\int_{0}^{\infty} \frac{d a(s)}{s+t} .
$$

Для дискретных мер $d a(s)=\sum a_{j} \delta\left(s-s_{j}\right) d s$ имеем $g(d a, t)=\sum a_{j} /\left(s_{j}+t\right)$, так что ступенчатая функция $u(y), y \in(0, Y)$, тогда и только тогда принадлежит множеству $\mathscr{U}_{k}$, когда

$$
\sum_{j=1}^{k} \frac{a_{j}}{s_{j}+t} \leqslant M(t), \quad t \in[0,1] .
$$

Множество $\mathscr{A}_{k}$. Соответствуюшее функциям из $\mathscr{U}_{k}$ множество дискретных неотрицательных мер

$$
\left\{\sum_{j=1}^{k} a_{j} \delta\left(s-s_{j}\right) d s \mid a_{j} \geqslant 0,1 \leqslant j \leqslant k ; \sum_{j=1}^{k} a_{j}=1 ; s_{1}>\cdots>s_{k}>0\right\},
$$

сосредоточенных не более чем в $k$ точках на полуоси $s>0$ и удовлетворяющих неравенству (36), обозначим через $\mathscr{A}_{k}$.

Семейство задач о верхней грани функционала $h(d a, t)$. Представим функцию $\kappa(s, t)$ из $(28)$ (как в $\S 2)$ в виде

$$
\kappa(s, t)=\frac{2}{\pi} \int_{t}^{\infty} \frac{d \sqrt{x}}{s+x}, \quad s>0, \quad t \geqslant 0 .
$$

Для функций $u(y) \in \mathscr{U}$ или, что то же, на множестве $\mathscr{A}$ тех неотрицательных мер $d a=d a(s)$, для которых (см. (29), (34) и (35))

$$
\int_{0}^{\infty} d a=1 \text { и } g(d a, x)=\int_{0}^{\infty} \frac{d a(s)}{s+x} \leqslant M(x) \text { при } x \in[0,1],
$$

рассмотрим однопараметрическое (зависящее от $t$ ) семейство экстремальных задач: найти верхнюю грань функционала

$$
h(d a, t)=\int_{0}^{\infty} \kappa(s, t) d a(s)=\frac{2}{\pi} \int_{t}^{\infty} g(d a, x) d \sqrt{x}, \quad 0<t<1 .
$$

Ввиду (23) и $(29) \sup h(d a, t)$ по множеству (38) - это ширина полосы $G$ вдоль прямой $u=1 / p=1 / \sqrt{1-t}$, так что теоремы $1-5$ будут доказаны, если проверить, что верны следующие утверждения. 
УТВЕРЖДЕНИЕ 1. Пусть $\sqrt{t_{k}}$ - наименьший положительный корень полинома Лежандра $L_{2 k+1}, k \geqslant 1$. Тогда при любом $t \geqslant t_{k} \sup h(d a, t)$ по множеству $\mathscr{A}$ мер (38) совпадает $c \sup h(d a, t)$ nо $\mathscr{A}_{k}$.

УТВЕРЖДЕНИЕ 2. При любом $t^{0} \geqslant t_{k}$ существуют такая мера $d a^{0} \in \mathscr{A}_{k}$, что при $d a=d a^{0}$ функционал $h\left(d a, t^{0}\right)$ достигает своего максимального значения $h^{0}$ на множестве $\mathscr{A}$ мер (38). Для любой мерь $d a$ из $\mathscr{A}$, отличной от $d a^{0}, h\left(d a, t^{0}\right)$ строго меньше $h^{0}$.

Мы докажем утверждение 1 в $\S 6$ и $\S 7$ и утверждение 2 - в $\S 8$ и $\S 9$, а в заключение этого параграфа сформулируем еше две теоремы о функциях $u_{P}(y)$ (которые докажем в $\S 9$ и 110$)$.

ТЕОРема 6. При любом $\varepsilon>0$, если координата и точки $P=(y, u)$ нижней границы полосы $G$ больше $1+\varepsilon$, то либо $n_{P}=1$ (т.е. функция и $P(y)$ принимает в волноводе лишь одно значение, не равное 1), либо значения $u_{P}(y)$ отделены от нуля константой порядка $\sqrt{\varepsilon}$, не зависящей от $\tau(p)$.

ОПРЕДЕЛЕНИЕ. Для каждой скоростной функции $u(y) \in \mathscr{U}$ (или, что то же, для каждой меры $d a \in \mathscr{A})$ точки $q \in[0,1]$, в которых $-\psi^{\prime}(q)=m(q)$, и, соответственно, точки $t \in[0,1]$, в которых $g(d a, t)=M(t)$, назовем особъми точками и (у) (или особъми точками $d a$ ).

С учетом (30), (31) геометрический смысл данного определения таков: для особых точек $q \in \mathscr{C}$ из интервала $(0,1)$ касательная к графику $u(y)$ в точке $\left(y, u=1 / \sqrt{1-q^{2}}\right)$ параллельна оси и.

ТЕОРема 7. Функция и ${ }_{P}(y)$ имеет минимум $n_{P}$ особъх точек (т.е. число особых точек не меньше числа не равных 1 значений $u_{P}(y)$ в волноводе).

Теоремы 1-7 - шаг к решению задачи, не поддававшейся старьм методам: $\partial л я$ произвольно заданной скоростной функции и(у) построить с заданной точностью множество $G$, заметаемое графиками всех скоростных функций, которые имеют тот же годограф, что и и(у).

\section{§6. Что используется в доказательстве теорем об универсальной последовательности?}

Две задачи. При проверке утверждения 1, эквивалентного теоремам 1,2 об универсальной последовательности, перед нами возникнут (вспомним $\left.{ }^{\langle 2\rangle}\right)$ следующие две задачи:

ЗАДАчА 1. Найти $c_{1}, \ldots, c_{n}$ и $\zeta_{1}, \ldots, \zeta_{n}$, удовлетворяющие нелинейной системе уравнений

$$
\sum_{j=1}^{n} c_{j} \zeta_{j}^{k}=\frac{1}{2 k+3}, \quad 0 \leqslant k \leqslant 2 n-1 .
$$


ЗАДАЧА 2. Найти такие $2 n$ чисел $c_{1}, \ldots, c_{n} u \zeta_{1}, \ldots, \zeta_{n}$, чтобъ для любого многочлена $\mathscr{P}(t)$ степени $k \leqslant 2 n-1$ и для функиии $\nu(t)=t^{3 / 2} / 3$ выполнялись равенства

$$
\int_{0}^{1} \mathscr{P}(t) d \nu(t)=\sum_{j=1}^{n} c_{j} \mathscr{P}\left(\zeta_{j}\right)
$$

Рассуждая в духе [15], нетрудно доказать ${ }^{\langle 14\rangle}$, что верна следующая

Теорема 8. Задачи 1 и 2 әквивалентны друг другу. Их решение существует и единственно, причем $\zeta_{1}, \ldots, \zeta_{n}$ - это квадраты ненулевых корней полинома Лежандра $L_{2 n+1}(x)$, a $c_{1}, \ldots, c_{n}$ определяются из системы линейных уравнений

$$
\sum_{j=1}^{n} c_{j} \zeta_{j}^{k}=\frac{1}{2 k+3}, \quad 0 \leqslant k \leqslant n-1 .
$$

Наряду с теоремой 8 при проверке утверждения 1 используются некоторые (приводимые ниже) результаты, полученные в [2].

Рациональные аппроксимации $r(\mathscr{S}, s)$. Произвольно фиксируем в $(37)$ какое-нибудь $t>0$ и положим $\varphi(s)=\kappa(s, t)$.

В соответствии с [2] сопоставим каждому набору точек

$$
\mathscr{S}=\left\{s_{1}, \ldots, s_{m+1}\right\}, \quad s_{1}>\cdots>s_{m+1}>0,
$$

такую рациональную аппроксимацию

$$
r(\mathscr{S}, s)=d_{0}+\sum_{k=1}^{m} \frac{d_{k}}{s+w_{k}}+\frac{d_{m+1}}{s}
$$

функции $\varphi(s)$, что

$$
r\left(\mathscr{S}, s_{j}\right)=\varphi\left(s_{j}\right), \quad r^{\prime}\left(\mathscr{S}, s_{j}\right)=\varphi^{\prime}\left(s_{j}\right), \quad 1 \leqslant j \leqslant m+1 .
$$

Согласно [8] такая рациональная аппроксимация существует, однозначно ( с точностью до нумерачии слагаемых) определяется условиями (42) и обладает следующими свойствами:

1) все полюсы $w_{k}, 1 \leqslant k \leqslant m$, в (41) различньи и положительньи, так что $r(\mathscr{S}, s)$ можно записать в виде

$$
d_{0}+\sum_{k=1}^{m+1} \frac{d_{k}}{s+w_{k}}
$$

u считать, что

$$
w_{1}>w_{2}>\cdots>w_{m+1}=0 ;
$$

2) все коэффициенты $d_{k}$ положсительны: $d_{k}>0,0 \leqslant k \leqslant m+1$;

3) при всех $s>0, s \neq s_{1}, \ldots, s_{m+1}$ выполняется строгое неравенство

$$
r(\mathscr{S}, s)>\varphi(s) \text {. }
$$

Согласно [2] рациональные аппроксимации $r(\mathscr{S}, s)$ позволяют следующим образом классифицировать дискретные меры $d a$. 
Классификация дискретных мер. Произвольно фиксируем $n \geqslant 1$ и дискретную меру

$$
d a^{0}=d a^{0}(s)=\sum_{j=1}^{n} a_{j}^{0} \delta\left(s-s_{j}^{0}\right) d s,
$$

сосредоточенную в $n$ точках:

$$
a_{j}^{0}>0, \quad 1 \leqslant j \leqslant n ; \quad \sum_{j=1}^{n} a_{j}^{0}=1 ; \quad s_{1}^{0}>\cdots>s_{n}^{0}>0 .
$$

При всевозможных натуральных $k$ возьмем все дискретные меры

$$
\begin{gathered}
d a=d a(s)=\sum_{j=1}^{k} a_{j} \delta\left(s-s_{j}\right) d s \\
a_{j}>0, \quad 1 \leqslant j \leqslant k ; \quad \sum_{j=1}^{k} a_{j}=1 ; \quad s_{1}>\cdots>s_{k}>0
\end{gathered}
$$

для которых (ср. с (36))

$$
g(d a, t)=\sum_{j=1}^{k} \frac{a_{j}}{s_{j}+t} \leqslant g\left(d a^{0}, t\right)=\sum_{j=1}^{n} \frac{a_{j}^{0}}{s_{j}^{0}+t}, \quad t \in[0,1],
$$

и рассмотрим вспомогательную задачу: найти максимум функционала $h(d a, t)$ из (39) по всем таким мерам. В [2] доказано, что решение этой задачи существует и единственно: на некоторой мере $d a$ из (46) $h(d a, t)$ достигает строгого максимума. При этом, если указанная мера

$$
\begin{gathered}
d a=d a(s)=\sum_{j=1}^{m+1} a_{j} \delta\left(s-s_{j}\right) d s \\
a_{j}>0, \quad 1 \leqslant j \leqslant m+1 ; \quad \sum_{j=1}^{m+1} a_{j}=1 ; \quad s_{1}>\cdots>s_{m+1}>0,
\end{gathered}
$$

сосредоточена в точках набора $\mathscr{S}$ (см. $(40),(41))$, то согласно [2] для полюса $w_{1}$ рациональной аппроксимации $r(\mathscr{S}, s)$ вьполняется неравенство $w_{1} \leqslant 1$.

Сформулируем этот важный для дальнейшего результат в виде леммы. Отнесем меру, сосредоточенную в точках (40) набора $\mathscr{S}$, к множествам $\mathscr{L}, \mathscr{E}$ и $\mathscr{G}$ (по первьм буквам английских слов less, equal и greater), если для полюса w $w_{1}$ annpoксимачии $r(\mathscr{S}, s)$ выполняется, соответственно, неравенство

$$
w_{1}<1, \quad w_{1}=1, \quad w_{1}>1
$$

Для мер $d a$ из (46) введем обозначение $d a \prec d a^{0}$ (это - отношение частичной упорядоченности на дискретных мерах). 
Лемма 1. Максимум $h(d a, t)$ по всем $d a \prec d a^{0}$ не может достигаться на мере $d a \in \mathscr{G}$. Он достигается на мере $d a \in \mathscr{M}=\mathscr{L} \cup \mathscr{E}$.

Чтобы использовать эту (доказанную в [2]) лемму для проверки утверждения 1 , введем еше один класс аппроксимаций функции $\varphi(s)$.

Рациональные аппроксимации $r_{0}(s)$. Определим аппроксимацию

$$
r_{0}(s)=d_{00}+\sum_{k=1}^{m} \frac{d_{0 k}}{s+w_{0 k}}
$$

функции $\varphi(s)$ (ср. с (42), (43)) условиями

$$
r_{0}^{(j)}(0)=\varphi^{(j)}(0), \quad 0 \leqslant j \leqslant 2 m^{\langle 15\rangle} .
$$

Нумерацию слагаемых в (47) выберем так, чтобы

$$
w_{01}=\max w_{0 k}, \quad 1 \leqslant k \leqslant m .
$$

Опираясь на (45), нетрудно доказать ${ }^{\langle 16\rangle}$, что верна

Лемма 2. Полюс $w_{1}$ в (44) больше, чем $w_{01}$ в (49):

$$
w_{1}>w_{01}
$$

Таким образом ${ }^{\langle 17\rangle}$, остается проверить

УТВЕРЖДЕНИЕ 3. Пусть $\sqrt{t_{m}}-$ наименьший положительный корень полинома Лежандра $L_{2 m+1}, m \geqslant 1$. Тогда при $t \geqslant t_{m}$ полюс $w_{01}$ аппроксимачии $r_{0}(s)$ функции $\varphi(s)=\kappa(s, t)$ не меньше 1 :

$$
w_{01} \geqslant 1
$$

\section{§7. Доказательство теорем об универсальной последовательности}

Возьмем $t=1$ в $(28)$ и $(37)$ и положим $\widehat{\varphi}(s)=\kappa(s, 1)$,

$$
\widehat{\varphi}(s)=\frac{2}{\pi} \frac{\operatorname{arctg} \sqrt{s}}{\sqrt{s}}=\frac{2}{\pi} \int_{1}^{\infty} \frac{d \sqrt{x}}{s+x}, \quad s \geqslant 0 .
$$

Пусть $\widehat{r}_{0}(s)$ - рациональная аппроксимация функции $\widehat{\varphi}(s)$, аналогичная $(47)$,

$$
\begin{aligned}
\widehat{r}_{0}(s) & =\widehat{d}_{00}+\sum_{k=1}^{m} \frac{\widehat{d}_{0 k}}{s+\widehat{w}_{0 k}}, \\
\widehat{r}_{0}^{(j)}(0) & =\widehat{\varphi}^{(j)}(0), \quad 0 \leqslant j \leqslant 2 m .
\end{aligned}
$$

Из (52) и (53) следует, что

$$
\widehat{\varphi}(0)=\frac{2}{\pi}, \quad \widehat{r}_{0}(0)=\widehat{d}_{00}+\sum_{k=1}^{m} \frac{\widehat{d}_{0 k}}{\widehat{w}_{0 k}}
$$


и что при $1 \leqslant j \leqslant 2 m$

$$
\frac{(-1)^{j}}{j !} \widehat{\varphi}^{(j)}(0)=\frac{2}{\pi} \int_{1}^{\infty} \frac{d \sqrt{x}}{x^{j+1}}, \quad \frac{(-1)^{j}}{j !} \widehat{r}^{(j)}(0)=\sum_{k=1}^{m} \frac{\widehat{d}_{0 k}}{\widehat{w}_{0 k}^{j+1}} .
$$

Поэтому условия (54) эквивалентны системе уравнений

$$
\begin{gathered}
\widehat{d}_{00}+\sum_{k=1}^{m} \frac{\widehat{d}_{0 k}}{\widehat{w}_{0 k}}=\frac{2}{\pi}, \\
\sum_{k=1}^{m} \frac{\widehat{d}_{0 k}}{\widehat{w}_{0 k}^{j+1}}=\frac{2}{\pi} \int_{1}^{\infty} \frac{d \sqrt{x}}{x^{j+1}}, \quad 1 \leqslant j \leqslant 2 m .
\end{gathered}
$$

Для доказательства утверждения 3 достаточно рассмотреть последние $2 m$ из этих уравнений. Если сделать замену

$$
\zeta_{k}=\frac{1}{\widehat{w}_{0 k}}, \quad c_{k}=\frac{\pi}{2} \frac{\widehat{d}_{0 k}}{\widehat{w}_{0 k}^{2}}, \quad 1 \leqslant k \leqslant m ; \quad t=\frac{1}{x}
$$

и ввести функцию $\nu(t)=t^{3 / 2} / 3$, они принимают вид

$$
\sum_{k=1}^{m} c_{k} \zeta_{k}^{p}=\int_{0}^{1} t^{p} d \nu(t), \quad 0 \leqslant p \leqslant 2 m-1 .
$$

Вычислив интегралы, получаем систему уравнений

$$
\sum_{k=1}^{m} c_{k} \zeta_{k}^{p}=\frac{1}{2 p+3}, \quad 0 \leqslant p \leqslant 2 m-1
$$

т.е. оказываемся в условиях теоремы 8 из $\S 6$. Занумеруем $\zeta_{k}$ в соответствии с (49) так, что $0<\zeta_{1}<\cdots<\zeta_{m}$, тогда (по теореме 8) $\zeta_{1}=t_{m}$ - квадрат наименьшего положительного корня полинома Лежандра $L_{2 m+1}$, т.е.

$$
\widehat{w}_{01}=\frac{1}{t_{m}} .
$$

Сравнивая (28) и (52), видим, что $\varphi(s)=\widehat{\varphi}(s / t) / \sqrt{t}$, и, значит, рациональные аппроксимации $r_{0}(s)$ и $\widehat{r}_{0}(s)$ из $(47)$ и $(53)$ связаны соотношением

$$
r_{0}(s)=\frac{\widehat{r}_{0}(s / t)}{\sqrt{t}},
$$

т.e.

$$
r_{0}(s)=\frac{\widehat{d}_{00}+\sum_{k=1}^{m} \widehat{d}_{0 k} /\left(s / t+\widehat{w}_{0 k}\right)}{\sqrt{t}}=\frac{\widehat{d}_{00}}{\sqrt{t}}+\sum_{k=1}^{m} \frac{\widehat{d}_{0 k} \sqrt{t}}{s+\widehat{w}_{0 k} t}
$$


и $w_{0 k}=\widehat{w}_{0 k} t, 1 \leqslant k \leqslant m$, откуда с учетом (55) $w_{01}=t / t_{m}$. Итак, $w_{01} \geqslant 1$ при $t \geqslant t_{m}$ и утверждение 3 доказано, а вместе с ним доказаны утверждение 1 и теоремы 1 и 2 .

\section{§ 8. Доказательство теоремы существования}

В этом параграфе будет доказана теорема 3 - существование скоростной функции $u_{P}(y)$, график которой проходит через произвольно заданную точку $P=(y, u), u>1$, нижней гранишы полосы $G$. Вернее, будет доказана уточняющая ее первая часть утверждения 2 из $\S 5$ - существование такой мерь $d a^{0} \in \mathscr{A}_{k}$, ито при $d a=d a^{0}$ функичонал $h\left(d a, t^{0}\right)$ достигает своего максимального значения $h^{0}$ на множестве $\mathscr{A}$ мер (38).

Множество $\tilde{\mathscr{A}}_{k}$. Множество дискретных неотрицательных мер

$$
\left\{\sum_{j=1}^{k} a_{j} \delta\left(s-s_{j}\right) d s \mid a_{j} \geqslant 0,1 \leqslant j \leqslant k ; \sum_{j=1}^{k} a_{j} \leqslant 1 ; s_{1}>\cdots>s_{k}>0\right\}
$$

сосредоточенных не более чем в $k$ точках на полуоси $s>0$ и удовлетворяющих неравенству (36), обозначим через $\widetilde{\mathscr{A}}_{k}$.

ЗАмечаниЕ. Определение $\tilde{\mathscr{A}}_{k}$ почти совпадает с определением $\mathscr{A}_{k}$ в $\S 5$, только в $\mathscr{A}_{k}$ включаются меры, для которых $\sum a_{j}=1$, а в $\mathscr{\mathscr { A }}_{k}-$ меры, для которых $\sum a_{j} \leqslant 1$.

Верхнюю грань $h(d a, t)$ по всем $d a \in \widetilde{\mathscr{A}}_{k}$ обозначим через $\tilde{h}_{k}(t)$, a sup $h(d a, t)$ по всем $d a \in \mathscr{A}_{k}$ - через $h_{k}(t), t \in[0,1]$.

ЛЕмма 3. При $t \geqslant t_{k}$ справедливо равенство $\tilde{h}_{k}(t)=h_{k}(t)$.

ДоказАтельство. Так как $\tilde{\mathscr{A}}_{k} \supset \mathscr{A}_{k}$, то $\tilde{h}_{k}(t) \geqslant h_{k}(t)$ при любом $t$. С другой стороны, для любой меры $d \tilde{a} \in \widetilde{\mathscr{A}}_{k}$ и любого $\varepsilon>0$ нетрудно найти такую меру $d a \in \mathscr{A}_{k+1}$, что $h(d a, t)>h(d \tilde{a}, t)-\varepsilon^{\langle 18\rangle}$, т.е. $h_{k+1}(t) \geqslant \tilde{h}_{k}(t)$. Но по утверждению 1 из $\S 5 h_{k}(t)=h_{k+1}(t)$, если $t \geqslant t_{k}$. Лемма доказана.

Мера $d a^{0}$. Пусть $t^{0} \geqslant t_{k}$. Возьмем последовательность мер

$$
d a_{n} \in \mathscr{A}_{k}, \quad d a_{n}=d a_{n}(s)=\sum_{j=1}^{k} a_{n j} \delta\left(s-s_{n j}\right) d s
$$

для которой

$$
h\left(d a_{n}, t^{0}\right) \rightarrow h_{k}\left(t^{0}\right) .
$$

Переходя, если нужно, к подпоследовательности, будем считать, что в (56) все $a_{n j}$ сходятся и все $s_{n j}$ имеют предел - конечный или бесконечный: при каждом $j$, $1 \leqslant j \leqslant k$

$$
a_{n j} \rightarrow a_{j}^{0} \geqslant 0, \quad s_{n j} \rightarrow s_{j}^{0} \geqslant 0 \text { или } s_{n j} \rightarrow+\infty
$$

Все индексы $j, 1 \leqslant j \leqslant k$, разобьем на три множества $-J_{0}, J_{\text {и }} J_{\infty}$ :

$$
j \in J_{0} \text { при } s_{n j} \rightarrow 0, \quad j \in J \text { при } s_{n j} \rightarrow s_{j}^{0}>0, \quad j \in J_{\infty} \text { при } s_{n j} \rightarrow+\infty \text {. }
$$


Суммируя $a_{j}^{0} \delta\left(s-s_{j}^{0}\right) d s$ по $j \in J$, получаем меру

$$
d a^{0}=\sum a_{j}^{0} \delta\left(s-s_{j}^{0}\right) d s \in \widetilde{\mathscr{A}}_{k},
$$

на которой ${ }^{\langle 19\rangle} h\left(d a^{0}, t^{0}\right)=h_{k}\left(t^{0}\right)=\sup h\left(d a, t^{0}\right)$ по всем $d a \in \mathscr{A}_{k}$. Докажем, что, на самом деле, построенная мера принадлежит $\mathscr{A}_{k}$. Пусть в (57) $m$ коэффициентов положительны. Изменим их нумерацию и обозначим их через

$$
a_{1}^{0}, \ldots, a_{m}^{0} .
$$

Лемма 4. СУмма $a_{1}^{0}+\cdots+a_{m}^{0}$ равна 1.

ДокАЗАТЕЛЬСтво. Допустив, что для меры $d a^{0}$

$$
a_{1}^{0}+\cdots+a_{m}^{0}=a<1,
$$

приведем это допущение к противоречию с тем, что по лемме 3

$$
h\left(d a^{0}, t^{0}\right)=\tilde{h}_{k}\left(t^{0}\right)=\tilde{h}_{k+1}\left(t^{0}\right) .
$$

Распространим на $\widetilde{\mathscr{A}}_{k}$ отношение частичной упорядоченности

$$
d a \prec d a^{0},
$$

введенное в $\S 6$ для мер из $\mathscr{A}_{k} ;$ пусть в соответствии с (46) для мер $d a$ и $d a^{0}$ из $\widetilde{\mathscr{A}}_{k}$ обозначение (60) означает, что

$$
g(d a, t)=\sum \frac{a_{j}}{s_{j}+t} \leqslant g\left(d a^{0}, t\right)=\sum \frac{a_{j}^{0}}{s_{j}^{0}+t}, \quad t \in[0,1] .
$$

Докажем, чтопривыполнении (58) можнопостроитьпринадлежашую $\tilde{\mathscr{A}}_{k}$ или $\tilde{\mathscr{A}}_{k+1}$ меру $d a \prec d a^{0}$, для которой вопреки (59)

$$
h\left(d a, t^{0}\right)>h\left(d a^{0}, t^{0}\right) .
$$

Если $s_{1}^{0} \geqslant 1$, то $d a \in \widetilde{\mathscr{A}}_{k}$ строится совсем просто ${ }^{\langle 20\rangle}$. При $s_{1}^{0}<1$ построение $d a \in \widetilde{\mathscr{A}}_{k+1}$ чуть сложнее (оно годится и для $s_{1}^{0} \geqslant 1$ ): сохраняя $a_{j}^{0}, s_{j}^{0}, 2 \leqslant j \leqslant m$, заменим пару $\left\{a_{1}^{0}, s_{1}^{0}\right\}$ в $d a^{0}$ четверкой $\left\{\delta, a_{1}^{0}-\varepsilon, S, s=s_{1}^{0}\right\}$ в $d a$, где $S>s$ и $\delta$ выбираются так, что

$$
(S+1) \varphi(S)>(s+1) \varphi(s), \quad \delta=\varepsilon \frac{S+1}{s+1},
$$

а $\varepsilon \in\left(0, a_{1}^{0}\right)$ столь мало, что

$$
\delta-\varepsilon=\varepsilon\left(\frac{S+1}{s+1}-1\right) \leqslant 1-a .
$$

Тогда

1) сумма коэффициентов в $d a$ равна $a+\delta-\varepsilon \leqslant 1$;

2) $d a \prec d a^{0}$, так как на отрезке $0 \leqslant x \leqslant 1$

$$
\begin{aligned}
g\left(d a^{0}, x\right)-g(d a, x) & =\frac{\varepsilon}{s+x}-\frac{\delta}{S+x}=\frac{\varepsilon}{s+1}\left(\frac{s+1}{s+x}-\frac{S+1}{S+x}\right) \geqslant 0 ; \\
\text { 3) } h\left(d a, t^{0}\right)-h\left(d a^{0}, t^{0}\right) & =\delta \varphi(S)-\varepsilon \varphi(s)=\frac{\varepsilon}{s+1}((S+1) \varphi(S)-(s+1) \varphi(s))>0 .
\end{aligned}
$$

Итак, неравенство (61), противоречащее (59), доказано, т.е. для мерь $d a^{0}$ в (57) сумма $a_{1}^{0}+\cdots+a_{m}^{0}$ равна 1 , и, значит, $d a^{0} \in \mathscr{A}_{k}$, что и требовалось установить ${ }^{\langle 21\rangle}$. 


\section{§9. Доказательство теоремы о строгом максимуме}

В $\S 8$ было доказано, что сушествует такая мера

$$
d a^{0}=d a^{0}(s)=\sum_{j=1}^{m} a_{j}^{0} \delta\left(s-s_{j}^{0}\right) d s, \quad a_{j}^{0}>0, \quad 1 \leqslant j \leqslant m,
$$

что при $d a=d a^{0}$ функционал

$$
h\left(d a, t^{0}\right)=\int_{0}^{\infty} \kappa\left(s, t^{0}\right) d a(s)=\int_{0}^{\infty} \varphi(s) d a(s)
$$

достигает своего максимального значения $h^{0}$ на множестве $\mathscr{A}$ неотрицательных мер $d a=d a(s)$, для которых

$$
\int_{0}^{\infty} d a=1, \quad g(d a, x)=\int_{0}^{\infty} \frac{d a(s)}{s+x} \leqslant M(x) \text { при } x \in[0,1] .
$$

Докажем, что для любой меры $d a \in \mathscr{A}\left(d a \neq d a^{0}\right)$ выполняется строгое неравенство

$$
h=h\left(d a, t^{0}\right)<h^{0}=h\left(d a^{0}, t^{0}\right),
$$

и тем самым докажем вторую часть утверждения 2 (о строгом максимуме), а вместе с ней - и теоремы 4 и 5 . Попутно будет также доказана теорема 7 об особых точках.

Дополнительное предположение. Вначале допустим (потом мы обоснуем это допушение), что множество особых точек меры $d a^{0}$

$$
\left\{x \in[0,1] \mid g^{0}(x)=M(x)\right\},
$$

где функция

$$
g^{0}(x)=g\left(d a^{0}, x\right)=\int_{0}^{\infty} \frac{d a^{0}(s)}{s+x}=\sum_{j=1}^{m} \frac{a_{j}^{0}}{s_{j}^{0}+x}
$$

совпадает с мажорантой $M(x)$, состоит менее, чем из $2 m$ точек, и докажем неравенство (64) при этом дополнительном предположении.

Примем на веру одно утверждение, которое проверим позже.

УТВЕРЖДДЕНИЕ 4. Пусть мера $d a=d a^{0}(s)=\sum_{j=1}^{m} a_{j}^{0} \delta\left(s-s_{j}^{0}\right) d s$, на которой функционал

$$
h\left(d a, t^{0}\right)=\int_{0}^{\infty} \kappa\left(s, t^{0}\right) d a(s)=\int_{0}^{\infty} \varphi(s) d a(s)
$$

достигает своего максимального значения $h^{0}$ на множестве $\mathscr{A}$ мер (63), имеет менее $2 m$ особых точек. Обозначим их

$$
x_{1}, \ldots, x_{p}, \quad p<2 m .
$$

Тогда существуют такие неотрицательнье числа $d_{k}, 0 \leqslant k \leqslant p$, что функиия $r(s)=d_{0}+\sum_{k=1}^{p} d_{k} /\left(s+x_{k}\right)$ совпадает с $\varphi(s)$ nрu $s=s_{j}^{0}, 1 \leqslant j \leqslant m$, а в остальных $s>0$ не меньие $\varphi(s)$. 
ЗАмечАниЕ 1 . Из утверждения 4 легко следует ${ }^{\langle 22\rangle}$, что

1) $r(s)=\varphi(s)$ в конечном числе точек $s_{1}^{0}, \ldots, s_{q}^{0}$, где $m \leqslant q \leqslant p$,

2) среди коэффициентов $d_{1}, \ldots, d_{p}$ имеется не менее $q$ положительных; пусть для определенности $d_{1}, \ldots, d_{q}>0, d_{q+1}, \ldots, d_{p} \geqslant 0$.

ЗАмечание 2. Из неравенства $p \geqslant m$ (см. замечание 1 ), очевидно, вытекает, что теорема 7 об особых точках является следствием утверждения 4 .

Доказательство (64) начнем с того, что в соответствии с (62), (63) и утверждением 4 преобразуем $h^{0}=h\left(d a^{0}, t^{0}\right)$, а затем оценим $h=h\left(d a, t^{0}\right)$ сверху

$$
\begin{aligned}
h^{0} & =\int_{0}^{\infty} \varphi(s) d a^{0}(s)=\sum_{j=1}^{m} a_{j}^{0} \varphi\left(s_{j}^{0}\right)=\sum_{j=1}^{m} a_{j}^{0} r\left(s_{j}^{0}\right)=\sum_{j=1}^{m} a_{j}^{0}\left(d_{0}+\sum_{k=1}^{p} \frac{d_{k}}{s_{j}^{0}+x_{k}}\right) \\
& =d_{0}+\sum_{k=1}^{p} d_{k} \sum_{j=1}^{m} \frac{a_{j}^{0}}{s_{j}^{0}+x_{k}}=d_{0}+\sum_{k=1}^{p} d_{k} g\left(d a^{0}, x_{k}\right)=d_{0}+\sum_{k=1}^{p} d_{k} g^{0}\left(x_{k}\right), \quad(67) \\
h & =\int_{0}^{\infty} \varphi(s) d a(s) \leqslant \int_{0}^{\infty} r(s) d a(s)=\int_{0}^{\infty}\left(d_{0}+\sum_{k=1}^{p} \frac{d_{k}}{s+x_{k}}\right) d a(s) \\
& =d_{0}+\sum_{k=1}^{p} d_{k} \int_{0}^{\infty} \frac{d a(s)}{s+x_{k}}=d_{0}+\sum_{k=1}^{p} d_{k} g\left(d a, x_{k}\right) \leqslant d_{0}+\sum_{k=1}^{p} d_{k} M\left(x_{k}\right) .
\end{aligned}
$$

Ввиду (65) и (66) из (67) и (68) следует, что

$$
h\left(d a, t^{0}\right) \leqslant d_{0}+\sum_{k=1}^{p} d_{k} M\left(x_{k}\right)=d_{0}+\sum_{k=1}^{p} d_{k} g^{0}\left(x_{k}\right)=h\left(d a^{0}, t^{0}\right),
$$

причем равенство $h\left(d a, t^{0}\right)=h\left(d a^{0}, t^{0}\right)$ в (69) достигается только в том случае, когда

$$
\int_{0}^{\infty}(\varphi(s)-r(s)) d a(s)=0
$$

и когда (см. замечание 1 к утверждению 4)

$$
g\left(d a, x_{k}\right)=M\left(x_{k}\right)=g^{0}\left(x_{k}\right) \text { при всех } k, 1 \leqslant k \leqslant q .
$$

Равенство (70) означает, что мера $d a$ сосредоточена в точках, где $\varphi(s)=r(s)$, т.е. при $s=s_{j}^{0}, 1 \leqslant j \leqslant q$, так что

$$
d a(s)=\sum_{j=1}^{q} a_{j} \delta\left(s-s_{j}^{0}\right) d s, \quad g(d a, x)=\int_{0}^{\infty} \frac{d a(s)}{s+x}=\sum_{j=1}^{q} \frac{a_{j}}{s_{j}^{0}+x},
$$

а ввиду (71) разность $g(d a, x)-g^{0}(x)$ имеет не менее $q$ корней

$$
g\left(d a, x_{k}\right)-g^{0}\left(x_{k}\right)=\sum_{j=1}^{q} \frac{a_{j}-a_{j}^{0}}{s_{j}^{0}+x_{k}}=0, \quad 1 \leqslant k \leqslant q^{\langle 23\rangle} .
$$


Однако, так как $d a \neq d a^{0}$,

$$
g(d a, x)-g^{0}(x)=\sum_{j=1}^{q} \frac{a_{j}-a_{j}^{0}}{s_{j}^{0}+x}
$$

не может иметь более $q-1$ корней (поскольку функции $1 /\left(s_{j}^{0}+x\right), 1 \leqslant j \leqslant q$, образуют чебышёвскую систему).

Итак, для завершения доказательства остается проверить утверждение 4 и обосновать допущение о том, что в (66) $p<2 m$.

Доказательство утверждения 4. Каждой мере $d a=d a(s) \in \mathscr{A}_{k}$,

$$
\begin{gathered}
d a(s)=\sum_{j=1}^{k} a_{j} \delta\left(s-s_{j}\right) d s \\
a_{j} \geqslant 0, \quad 1 \leqslant j \leqslant k ; \quad \sum_{j=1}^{k} a_{j}=1 ; \quad s_{1}>\cdots>s_{k}>0,
\end{gathered}
$$

сопоставим набор

$$
\mathscr{N}=\left\{a_{j}, s_{j}\right\}, \quad a_{j} \geqslant 0, \quad 1 \leqslant j \leqslant k, \quad s_{1}>\cdots>s_{k}>0
$$

и наряду с обозначениями $g(d a, x)$ и $h\left(d a, t^{0}\right)$ будем использовать обозначения $g(\mathscr{N}, x)$ и $h(\mathscr{N})$.

ЗАмечАнИЕ. Мы не включаем в (72) равенство $\sum_{j=1}^{k} a_{j}=1$, чтобы можно было рассматривать и такие наборы $\mathcal{N}$, в которых $\sum_{j=1}^{k} a_{j} \neq 1$.

Если в (66) $p<2 m-1$, добавим к $x_{1}, \ldots, x_{p}$ точки $x_{p+1}, \ldots, x_{2 m-1}$. При $p=2 m-1$ добавочные точки не нужны - хватает имеющихся. Положим

$$
r(s)=d_{0}+\sum_{k=1}^{2 m-1} \frac{d_{k}}{s+x_{k}}
$$

определив $d_{0}, \ldots, d_{2 m-1}$ из невырожденной ${ }^{\langle 24\rangle}$ системы линейных уравнений

$$
\varphi\left(s_{j}^{0}\right)=r\left(s_{j}^{0}\right), \quad \varphi^{\prime}\left(s_{j}^{0}\right)=r^{\prime}\left(s_{j}^{0}\right), \quad 1 \leqslant j \leqslant m .
$$

Чтобы проверить утверждение 4 , достаточно доказать, что

$$
\begin{gathered}
d_{1}, \ldots, d_{p} \geqslant 0, \\
d_{p+1}, \ldots, d_{2 m-1}=0, \\
\varphi(s) \leqslant r(s), \quad s>0 .
\end{gathered}
$$


ЗАмЕчАнИЕ. Неотрицательность $d_{0}$ следует из (76): так как $r(s) \geqslant \varphi(s) \geqslant 0$ при $s>0$, то и $d_{0}=\lim _{s \rightarrow \infty} r(s) \geqslant 0$.

Соотношения (74)-(76) будут доказаны по одной схеме: допуская поочередно, что они неверны, мы каждый раз сумеем тогда сместиться из набора $\mathscr{N}^{0}=\left\{a_{j}^{0}, s_{j}^{0}\right\}$ (соответствуюшего мере $d a^{0} \in \mathscr{A}_{m}$ ) в такой набор $\mathscr{N}^{*}=\left\{a_{j}^{*}, s_{j}^{*}\right\}$ (соответствуюший мере $d a^{*}$ из $\mathscr{A}_{m}$ или из $\left.\mathscr{A}_{m+1}\right)$, что $h\left(\mathscr{N}^{*}\right)>h\left(\mathscr{N}^{0}\right)$, и тем самым придем к противоречию с тем, что

$$
h\left(\mathscr{N}^{0}\right)=h\left(d a^{0}, t^{0}\right)=\max h\left(d a, t^{0}\right) \text { по всем } d a \in \mathscr{A} .
$$

Проверка неравенств (74). При $k=m$ для наборов $\mathscr{N}$ в $(72)$ числа

$$
a(\mathscr{N})=\sum_{j=1}^{m} a_{j}, \quad g\left(\mathscr{N}, x_{1}\right), \ldots, g\left(\mathscr{N}, x_{2 m-1}\right)
$$

являются локальными координатами в окрестности $\mathscr{N}^{0<25>}$.

Допустив, что хоть один коэффициент в (74) отрицателен, произвольно фиксируем такие $\xi_{1}, \ldots, \xi_{p}>0$, что

$$
\sum_{k=1}^{p} \xi_{k} d_{k}=-1
$$

и для малых $\lambda>0$ рассмотрим такие наборы $\mathscr{N}^{*}=\mathscr{N}^{*}(\lambda)$, что

$$
\begin{gathered}
a\left(\mathscr{N}^{*}\right)=a\left(\mathscr{N}^{0}\right)=1, \quad g\left(\mathscr{N}^{0}, x_{k}\right)-g\left(\mathscr{N}^{*}, x_{k}\right)=\lambda \xi_{k} \text { при } 1 \leqslant k \leqslant p \\
g\left(\mathscr{N}^{0}, x_{k}\right)=g\left(\mathscr{N}^{*}, x_{k}\right) \text { при } p<k \leqslant 2 m-1 .
\end{gathered}
$$

Иньми словами, мы сдвигаемся из $\mathscr{N}^{0}$ по лучу

$$
\mathscr{N}^{*}=\mathscr{N}^{*}(\lambda)=\mathscr{N}^{0}-\lambda \sum_{k=1}^{p} \xi_{k} \mathscr{E}_{k}, \quad \lambda>0,
$$

где $\mathscr{E}_{k}, 0 \leqslant k \leqslant 2 m-1,-$ орты в координатах (78).

Нетрудно проверить ${ }^{\langle 26\rangle}$, что для некоторого $\varepsilon>0$ и для достаточно мальх $\lambda$ при всех $x \in[0,1]$, принадлежащих є-окрестности

$$
V=\left(x_{1}-\varepsilon, x_{1}+\varepsilon\right) \cup \cdots \cup\left(x_{p}-\varepsilon, x_{p}+\varepsilon\right)
$$

множества точек $x_{1}, \ldots, x_{p}$, выполняется неравенство $g\left(\mathscr{N}^{*}, x\right)<g\left(\mathscr{N}^{0}, x\right)$.

Отсюда следует ${ }^{\langle 27\rangle}$, что при малых $\lambda$ на луче (82) верна

Лемма 5. При всех $x \in[0,1] \quad g\left(\mathscr{N}^{*}, x\right)<M(x)$, maк что набор $\mathscr{N}^{*}$ coomветствует мере $d a^{*} \in \mathscr{A}_{m}$.

При мальх $\lambda$ на луче (82) верна также ${ }^{\langle 28\rangle}$

Лемма 6. Справедливо неравенство $h\left(\mathscr{N}^{*}\right)>h\left(\mathscr{N}^{0}\right)$.

Полученное противоречие с (77) доказывает неравенства (74). 
Проверка равенств $(75)$. Если хоть один коэффицциент $d_{k}$ в $(75)$ не равен нулю, рассмотрим такие $\xi_{1}, \ldots, \xi_{p}>0$ и такое $\xi_{k}$, что

$$
\xi_{1} d_{1}+\cdots+\xi_{p} d_{p}+\xi_{k} d_{k}=-1
$$

и сдвинемся из $\mathscr{N}^{0}$ по лучу

$$
\mathscr{N}^{*}=\mathscr{N}^{*}(\lambda)=\mathscr{N}^{0}-\lambda\left(\xi_{1} \mathscr{E}_{1}+\cdots+\xi_{p} \mathscr{E}_{p}+\xi_{k} \mathscr{E}_{k}\right), \quad \lambda>0
$$

Рассуждая далее так же, как при проверке (74), приходим к выводу: при малых $\lambda$ на луче (84), как и на луче (82), набор $\mathscr{N}^{*}(\lambda)$ соответствует мере $d a^{*} \in \mathscr{A}_{m}$ $u h\left(\mathscr{N}^{*}\right)>h\left(\mathscr{N}^{0}\right)$, т.е. снова приходим к противоречию с (77).

Проверка неравенства (76). Допустим, что (76) не вьполняется: пусть

$$
\varphi(s)>r(s) \text { при } s=\bar{s}>0 .
$$

Положим $\bar{a}=0$, перепишем $d a^{0}$ в виде

$$
d a^{0}(s)=\sum_{j=1}^{m} a_{j}^{0} \delta\left(s-s_{j}^{0}\right) d s+\bar{a} \delta(s-\bar{s}) d s
$$

и в окрестности соответствующего $d a^{0}$ набора

$$
\mathscr{N}^{0}=\left\{a_{1}, \ldots, a_{m}, \bar{a}=0, s_{1}, \ldots, s_{m}, \bar{s}\right\}
$$

рассмотрим новые локальные координаты, добавив к (78) $\bar{a}$ и $\bar{s}$. Пусть $\overline{\mathscr{E}}-$ орт в этих координатах, соответствуюший координате $\bar{a}$, и пусть положительные числа $\xi_{0}, \xi_{1}, \ldots, \xi_{p}$ таковы, что

$$
\sum_{k=1}^{p} \xi_{k} d_{k}-\xi_{0}(\varphi(\bar{s})-r(\bar{s}))=-1
$$

Тогда при малых $\lambda$ на луче

$$
\mathscr{N}^{*}=\mathscr{N}^{*}(\lambda)=\mathscr{N}^{0}-\lambda\left(\sum_{k=0}^{p} \xi_{k} \mathscr{E}_{k}-\xi_{0} \overline{\mathscr{E}}\right), \quad \lambda>0
$$

верны модификация леммы 5 и лемма $6: g\left(\mathscr{N}^{*}, x\right)<M(x)$ при всех $x \in[0,1]$, так что набор $\mathscr{N}^{*}$ соответствует мере $d a^{*} \in \mathscr{A}_{m+1}$, и (вопреки $\left.(77)\right) h\left(\mathscr{N}^{*}\right)>h\left(\mathscr{N}^{0}\right)$.

Проверка утверждения 4 закончена, и, чтобы завершить доказательство теоремы о строгом максимуме, остается обосновать допушение о том, что в (66) $p<2 m$. 
Обоснование дополнительного предположения. Разумеется, множество $\left\{x \in[0,1] \mid g^{0}(x)=M(x)\right\}$, где функция

$$
g^{0}(x)=g\left(d a^{0}, x\right)=\int_{0}^{\infty} \frac{d a^{0}(s)}{s+x}=\sum_{j=1}^{m} \frac{a_{j}^{0}}{s_{j}^{0}+x}
$$

совпадает с мажорантой $M(x)$, может содержать более, чем $2 m$ точек, и даже может совпадать со всем отрезком $[0,1]$.

Почему же достаточно доказать (64) при дополнительном предположении, что это множество состоит из точек $x_{1}, \ldots, x_{p}$, где $p<2 m$ ?

Объяснение заключается в следующем. Допустим, что максимум $h^{0}$ функционала $h\left(d a, t^{0}\right)$ на множестве $\mathscr{A}($ см. (63)) достигается не только на мере

$$
d a^{0}(s)=\sum_{j=1}^{m} a_{j}^{0} \delta\left(s-s_{j}^{0}\right) d s \in \mathscr{A}
$$

но еще и на некоторой другой мере $d a^{1} \in \mathscr{A}$,

$$
\int_{0}^{\infty} d a^{1}=1, \quad g^{1}(x)=g\left(d a^{1}, x\right)=\int_{0}^{\infty} \frac{d a^{1}(s)}{s+x} \leqslant M(x) \text { при } x \in[0,1] .
$$

Воспользуемся следующей очевидной леммой $о$ выпуклости:

Лемма 7. Пусть меры $d a^{0}$ и $d a^{1}$ принадлежат множеству $\mathscr{A} u$

$$
h\left(d a^{0}, t^{0}\right)=h\left(d a^{1}, t^{0}\right)=h^{0},
$$

тогда при любых $\alpha^{0} \geqslant 0, \alpha^{1} \geqslant 0, \alpha^{0}+\alpha^{1}=1$, мера $d a=\alpha^{0} d a^{0}+\alpha^{1} d a^{1}$ mоже принадлежит множеству $\mathscr{A}$ и $h\left(d a, t^{0}\right)=h^{0}$.

Положим $d a^{*}=\frac{1}{2}\left(d a^{0}+d a^{1}\right), g^{*}(x)=\frac{1}{2}\left(g^{0}(x)+g^{1}(x)\right)$. Функция

$$
g^{*}(x)=g\left(d a^{*}, x\right)=\frac{1}{2}\left(\sum_{j=1}^{m} \frac{a_{j}^{0}}{s_{j}^{0}+x}+\int_{0}^{\infty} \frac{d a^{1}(s)}{s+x}\right)
$$

совпадает с мажорантой $M(x)$ в тех и только тех точках, где

$$
g^{0}(x)=g^{1}(x)=M(x),
$$

т.е. на подмножестве множества

$$
\left\{x \in[0,1] \mid \sum_{j=1}^{m} \frac{a_{j}^{0}}{s_{j}^{0}+x}=\int_{0}^{\infty} \frac{d a^{1}(s)}{s+x}\right\} .
$$

Но в множестве (85), очевидно, меньше, чем $2 m$ точек $^{\langle 29\rangle}$. Назовем точки

$$
\left\{x \in[0,1] \mid g^{0}(x)=g^{1}(x)=M(x)\right\}
$$


так же, как в (66),

$$
x_{1}, \ldots, x_{p}, \quad p<2 m,
$$

и перепишем неравенство

$$
g^{*}(x)=\frac{g^{0}(x)+g^{1}(x)}{2}<M(x) \text { при } x \in[0,1], \quad x \neq x_{1}, \ldots, x_{p}
$$

в виде

$$
g^{0}(x)<2 M(x)-g^{1}(x)=M^{*}(x) \text { при } x \in[0,1], \quad x \neq x_{1}, \ldots, x_{p} .
$$

Функцию $M^{*}(x)=2 M(x)-g^{1}(x)$ объявим новой мажсорантой. Через $\mathscr{A}^{*}$ обозначим множество тех мер $d a=d a(s)$, для которых

$$
\int_{0}^{\infty} d a=1 \quad \text { и } g(d a, x)=\int_{0}^{\infty} \frac{d a(s)}{s+x} \leqslant M^{*}(x) \text { при } x \in[0,1] .
$$

Сравнивая (86) и (63), легко получаем, что максимум функционала $h\left(d a, t^{0}\right)$ на множестве мер $d a \in \mathscr{A}^{*}$ равен $h^{0}$ и достигается на мерах $d a^{0}$ и $d a^{1}$.

Но по построению $g^{0}(x)=g\left(d a^{0}, x\right)=M^{*}(x)$ только в точках

$$
x_{1}, \ldots, x_{p}, \quad p<2 m,
$$

т.е. допушение о том, что $p<2 m$, выполняется. Итак, теорему о строгом максимуме, действительно, достаточно было доказать при этом дополнительном предположении.

Таким образом, если максимум функиионала $h\left(d a, t^{0}\right)$ достигается на меpax $d a^{0} u d a^{1} \in \mathscr{A}$, mo $d a^{1} \equiv d a^{0}$.

Доказательство теоремы о строгом максимуме завершено. Одновременно доказаны теоремы 4, 5, а еше раньше - вместе с утверждением 4 - доказана (ввиду замечания 2 к этому утверждению) и теорема 7.

\section{§10. Доказательство теоремы о минимуме скорости в волноводе}

Теперь докажем теорему 6 (см. конец $\S 5)$ о минимуме скоростной функции $u_{P}(y)$, график которой проходит через точку

$$
P=(y, u), \quad u>1+\varepsilon
$$

нижней границы полосы $G$. Пусть $n_{P}>1$ (т.е. $u_{P}(y)$ принимает в волноводе хотя бы два значения, не равных 1); проверим, что тогда

$$
\min u_{P}(y) \geqslant \sqrt{C \varepsilon}
$$

где $C$ - положительная константа, не зависящая от $\tau(p)$.

Ввиду (23), чтобы проверить (87) достаточно установить, что для соответствующей $u_{P}(y)$ меры

$$
d a=d a(s)=\sum_{j=1}^{m+1} a_{j} \delta\left(s-s_{j}\right) d s
$$


сосредоточенной в точках $s_{1}>\cdots>s_{m+1}>0$ (и такой, что все $a_{j}>0,1 \leqslant j \leqslant$ $\left.m+1, \sum_{j=1}^{m+1} a_{j}=1\right)$, максимальное из $s_{j}$ не может быть слишком больиим, а именно,

$$
s_{1} \leqslant \frac{1}{C \varepsilon}-1 .
$$

Вначале рассмотрим случай, когда в (88) $m=1$ :

$$
d a(s)=a_{1} \delta\left(s-s_{1}\right) d s+a_{2} \delta\left(s-s_{2}\right) d s, \quad s_{1}>s_{2}>0, a_{1,2}>0, \quad a_{1}+a_{2}=1 .
$$

В соответствии с $\S 6$ (см. (40), (41)) сопоставим набору точек $\mathscr{S}=\left\{s_{1}, s_{2}\right\}$ такую рациональную аппроксимацию

$$
r(\mathscr{S}, s)=d_{0}+\frac{d_{1}}{s+w_{1}}+\frac{d_{2}}{s}
$$

функции $\varphi(s)$, что $r\left(\mathscr{S}, s_{j}\right)=\varphi\left(s_{j}\right), r^{\prime}\left(\mathscr{S}, s_{j}\right)=\varphi^{\prime}\left(s_{j}\right), j=1,2$. Кроме того (ср. с (47), (48)), рассмотрим такую рациональную аппроксимацию

$$
r_{1}(s)=d_{10}+\frac{d_{11}}{s+w_{11}}
$$

функции $\varphi(s)$, что

$$
r_{1}(0)=\varphi(0), \quad r_{1}\left(s_{1}\right)=\varphi\left(s_{1}\right), \quad r_{1}^{\prime}\left(s_{1}\right)=\varphi^{\prime}\left(s_{1}\right)
$$

Нетрудно доказать ${ }^{\langle 30\rangle}$, что верна аналогичная лемме 2 из $\S 6$

ЛЕмма 8. Полюс $w_{11}$ аппроксимации $r_{1}(s)$ меньие, чем полюс $w_{1}$ аппроксимации $r(\mathscr{S}, s): w_{11}<w_{1}$.

А так как (по лемме 1$) w_{1} \leqslant 1$, то отсюда следует, что

$$
w_{11}<1
$$

и для установления (89) в случае $m=1$ остается доказать (это проверяется прямьм подсчетом $\left.{ }^{\langle 31\rangle}\right)$, что верна следующая

ЛЕМма 9. Пусть

$$
\varphi(s)=\frac{2}{\pi} \frac{\operatorname{arctg} \sqrt{s / t}}{\sqrt{s}}
$$

и пусть полюс $w_{11}$ аппроксимации $r_{1}(s)$ (см. (91), (92)) меньше 1. Тогда для некоторого $c>0$

$$
s_{1} \leqslant \frac{c}{t}
$$


ЗАмечаниЕ. Так как ввиду (23) координата $u$ точки $P$ равна $1 / \sqrt{1-t}$, неравенство (94) эквивалентно (89).

В обшем случае (для произвольного $m \geqslant 1$ ) вместо (90) нужно рассмотреть, как в (42)-(44), рациональную аппроксимацию

$$
\begin{gathered}
r(\mathscr{S}, s)=d_{0}+\sum_{k=1}^{m+1} \frac{d_{k}}{s+w_{k}}, \quad w_{1}>w_{2}>\cdots>w_{m+1}=0, \\
r\left(\mathscr{S}, s_{j}\right)=\varphi\left(s_{j}\right), \quad r^{\prime}\left(\mathscr{S}, s_{j}\right)=\varphi^{\prime}\left(s_{j}\right), \quad 1 \leqslant j \leqslant m+1,
\end{gathered}
$$

а вместо (91), (92) - рациональную аппроксимацию

$$
\begin{gathered}
r_{m}(s)=d_{m 0}+\sum_{k=1}^{m} \frac{d_{m k}}{s+w_{m k}}, \quad w_{m 1}>w_{m 2}>\cdots>w_{m m}>0 \\
r_{m}^{(k)}(0)=\varphi^{(k)}(0), \quad 0 \leqslant k \leqslant 2 m-2, \quad r_{m}\left(s_{1}\right)=\varphi\left(s_{1}\right), \quad r_{m}^{\prime}\left(s_{1}\right)=\varphi^{\prime}\left(s_{1}\right) .
\end{gathered}
$$

Для этих аппроксимаций выполняется ${ }^{\langle 32\rangle}$ следующий аналог леммы 8. Полюс $w_{m 1}$ аппроксимации $r_{m}(s)$ меньше, чем полюс $w_{1}$ аппроксимации $r(\mathscr{S}, s)$ :

$$
w_{m 1}<w_{1} .
$$

Следовательно, так как (по лемме 1$) w_{1} \leqslant 1$,

$$
w_{m 1}<1,
$$

и для завершения доказательства теоремы 6 остается заметить, что ${ }^{\langle 33\rangle} w_{m 1}$ в (95) при $m>1$ и $w_{11}$ в $(93)$ связаны неравенством $w_{11}<w_{m 1}$, позволяюшим и при $m>1$ применить лемму 9 .

\section{§11. Теорема о монотонности - формулировка и доказательство}

Теперь, вьполняя данное в $\S 2$ обешание, докажем (при некоторых дополнительных ограничениях) монотонность функции $n(t)$. А именно, докажем монотонность $n(t)$ для функционала (39) на множестве мер (38) в случае, детально исследованном в [2]: когда мажоранта $M(x), x \in[0,1]$, совпадает с функиией $g(d \hat{a}, x)$ для некоторой дискретной меры dâ. Подробнее: пусть

$$
\begin{gathered}
d \hat{a}=d \hat{a}(s)=\sum_{j=1}^{\widehat{n}} \hat{a}_{j} \delta\left(s-\widehat{s}_{j}\right) d s, \\
\hat{a}_{j}>0, \quad 1 \leqslant j \leqslant \widehat{n} ; \quad \sum_{j=1}^{\widehat{n}} \hat{a}_{j}=1 ; \quad \widehat{s}_{1}>\cdots>\widehat{s}_{\widehat{n}}>0,
\end{gathered}
$$

тогда относительно мажоранты $M(x)$ предполагается, что

$$
M(x)=g(d \hat{a}, x)=\int_{0}^{\infty} \frac{d \hat{a}(s)}{s+x}=\sum_{j=1}^{\widehat{n}} \frac{\hat{a}_{j}}{\widehat{s}_{j}+x}, \quad x \in[0,1]^{\langle 34\rangle} .
$$


С учетом [1]-[3] в случае (96) при

$$
\kappa(s, t)=\frac{2}{\pi} \int_{t}^{\infty} \frac{d \sqrt{x}}{s+x}=\frac{2}{\pi} \frac{\operatorname{arctg} \sqrt{s / t}}{\sqrt{s}}, \quad t \in(0,1] ; \quad \kappa(s, 0)=\frac{1}{\sqrt{s}},
$$

функционал

$$
h(d a, t)=\int_{0}^{\infty} \kappa(s, t) d a(s)=\frac{2}{\pi} \int_{t}^{\infty} g(d a, x) d \sqrt{x}, \quad 0 \leqslant t \leqslant 1,
$$

достигает максимума при каждом $t \in[0,1]$ на некоторой (зависящей от $\mathrm{t}$ ) дискретной мере $d a \prec d \hat{a}$, сосредоточенной в $n(t) \leqslant \widehat{n}$ точках, т.е. $\max h(d a, t)$ достаточно искать на мерах

$$
d a \in \mathscr{A}_{k}, \quad k \leqslant \widehat{n} .
$$

Рассмотрим два случая: основной, когда при любом $t \in[0,1]$ максимум $h(d a, t)$ достигается не на мере $d \hat{a}$, и исключительный, когда (при некоторых $t \in[0,1])$ $\max h(d a, t)=h(d \hat{a}, t)$.

Основной случай. Согласно [2] в этом случае (ср. с леммой 1 в $\S 6$ и утверждением 4 в $\S 9)$ верно следуюшее

УТВеРЖДЕНИЕ 5. Для каждого $t \in[0,1]$ существуют и однозначно определяются такое $n=n(t) \leqslant \widehat{n}$, такой набор чисел

$$
\begin{gathered}
\left\{a_{1}, \ldots, a_{n}, s_{1}, \ldots, s_{n}\right\}, \quad a_{j}=a_{j}(t)>0, \quad 1 \leqslant j \leqslant n, \quad \sum_{j=1}^{n} a_{j}=1, \\
s_{1}=s_{1}(t)>\cdots>s_{n}=s_{n}(t)>0,
\end{gathered}
$$

и такой набор чисел

$$
\begin{gathered}
\left\{b_{0}, b_{1}, \ldots, b_{n}, x_{1}, \ldots, x_{n}\right\}, \quad b_{k}=b_{k}(t)>0, \quad 0 \leqslant k \leqslant n, \\
1=x_{1}=x_{1}(t)>\cdots>x_{n}=x_{n}(t) \geqslant 0,
\end{gathered}
$$

чmo

1) строгий максимум функционала $h(d a, t)$ достигается на мере

$$
d a=d a(s, t)=\sum_{j=1}^{n} a_{j}(t) \delta\left(s-s_{j}(t)\right) d s, \quad 1 \leqslant j \leqslant n=n(t) ;
$$

2) функция

$$
g(d a, x)=\sum_{j=1}^{n} \frac{a_{j}(t)}{s_{j}(t)+x}
$$

совпадает с мажорантой $M(x)=g($ dâ, $x)$ в точках (100) и только в них, т.е. $\left\{x_{k}(t)\right\}_{k=1}^{n}$ - это множество особых точек меры (101);

3) функиия

$$
r(s, t)=b_{0}(t)+\sum_{k=1}^{n} \frac{b_{k}(t)}{s+x_{k}(t)}
$$

совпадает с $\kappa(s, t)$ из (97) в точках (99), а в остальных $s>0$ больwe $\kappa(s, t)$.

Верны также следующие два утверждения ${ }^{\langle 35\rangle}$. 
УТВЕРЖДЕНИЕ 6. Если $t_{i} \in[0,1]$ сходятся $\kappa$, mo $n\left(t_{i}\right) \geqslant n(t)$, начиная $c$ некоторого $i$, и (см. (99) и (100))

$$
\begin{array}{llrl}
s_{k}\left(t_{i}\right) & \rightarrow s_{k}(t) n p u k \leqslant n(t), & s_{k}\left(t_{i}\right) \rightarrow 0 \text { nрu } k>n(t) \\
x_{k}\left(t_{i}\right) \rightarrow x_{k}(t) n p u k \leqslant n(t), & x_{k}\left(t_{i}\right) \rightarrow 0 \text { nрu } k>n(t) .
\end{array}
$$

УТВЕРЖДЕНИЕ 7. Пусть $t>0-$ точка разрыва $n(t)$. Тогда

$$
0<t<x_{n}(t)
$$

Монотонность функции $n(t)$ является следствием этих утверждений: верна

Теорема 9. При условии (96) $n(t)$ не возрастает на $(0,1]$.

ДокАЗАТЕЛЬСтво. Пусть $t>0$ - точка разрьва функции $n(t)$. Тогда (с учетом утверждения 6) в любой ее окрестности есть точка $t^{*}$, в которой $n\left(t^{*}\right)>n(t)$. Положим (см. (101))

$$
d a=d a(s, t), \quad d a^{*}=d a\left(s, t^{*}\right), \quad g(x)=g(d a, x), \quad g^{*}(x)=g\left(d a^{*}, x\right) .
$$

Пусть точка $t^{*}$ настолько близка к $t$, что в соответствии с утверждениями 6 и 7 вьполняются условия

$$
\begin{gathered}
0 \leqslant x_{n+1}\left(t^{*}\right)<\min \left(t, t^{*}\right), \quad \max \left(t, t^{*}\right)<\min \left(x_{n}(t), x_{n}\left(t^{*}\right)\right) \\
0<s_{n+1}\left(t^{*}\right)<s_{n}(t) .
\end{gathered}
$$

Выведем из (103) и (104), что

$$
t^{*}<t
$$

а значит, $n(t)$ не возрастает ${ }^{\langle 36\rangle}$.

Проверка неравенства (105). Введем две функции:

$$
\begin{aligned}
P_{1}(x) & =g^{*}(x)-g(x)=\sum_{j=1}^{n\left(t^{*}\right)} \frac{a_{j}^{*}}{s_{j}^{*}+x}-\sum_{j=1}^{n(t)} \frac{a_{j}}{s_{j}+x} \\
P_{2}(s) & =\left(r\left(s, t^{*}\right)-\kappa\left(s, t^{*}\right)\right)-(r(s, t)-\kappa(s, t))=r\left(s, t^{*}\right)-r(s, t)+\frac{2}{\pi} \int_{t}^{t^{*}} \frac{d \sqrt{x}}{s+x} \\
& =b_{0}\left(t^{*}\right)-b_{0}(t)+\sum_{j=1}^{n\left(t^{*}\right)} \frac{b_{k}\left(t^{*}\right)}{s+x_{k}\left(t^{*}\right)}-\sum_{j=1}^{n(t)} \frac{b_{k}(t)}{s+x_{k}(t)}+\frac{2}{\pi} \int_{t}^{t^{*}} \frac{d \sqrt{x}}{s+x}
\end{aligned}
$$

Приведя подобные члены, запишем $P_{1}(x)$ и $P_{2}(s)$ в виде

$$
P_{1}(x)=\sum_{j=1}^{N} \frac{A_{j}}{S_{j}+x}, \quad P_{2}(s)=B_{0}+\sum_{k=1}^{Q} \frac{B_{k}}{s+X_{k}}+\frac{2}{\pi} \int_{t}^{t^{*}} \frac{d \sqrt{x}}{s+x},
$$

где $S_{1}>\cdots>S_{N}, X_{1}>\cdots>X_{Q}$. 
Так же, как в [3, с. 23, 24], доопределим ядро Коши $C(s, x)=1 /(s+x)$ при $x=X_{0}=+\infty$, положив $C\left(s, X_{0}\right)=C(s,+\infty)=1$. Тогда формулы для $P_{1}(x)$ и $P_{2}(s)$ примут вид

$$
P_{1}(x)=\sum_{j=1}^{N} A_{j} C\left(S_{j}, x\right), \quad P_{2}(s)=\sum_{k=0}^{Q} B_{k} C\left(s, X_{k}\right)+\frac{2}{\pi} \int_{t}^{t^{*}} C(s, x) d \sqrt{x},
$$

причем (вследствие равенств $\left.\sum a_{j}=\sum a_{j}^{*}=1\right)$

$$
P_{1}\left(X_{0}\right)=P_{1}(+\infty)=0 .
$$

Выберем индекс $q$ так, чтобы $x_{n+1}\left(t^{*}\right)=X_{q+1}$, и рассмотрим следующие три последовательности:

$$
\begin{gathered}
\left\{P_{1}\left(X_{Q}\right), \ldots, P_{1}\left(X_{1}\right), P_{1}\left(X_{0}\right)\right\}, \quad\left\{P_{2}\left(S_{N}\right), \ldots, P_{2}\left(S_{1}\right)\right\} \\
\left\{P_{1}\left(X_{Q}\right), \ldots, P_{1}\left(X_{q+1}\right), t^{*}-t, P_{1}\left(X_{q}\right), \ldots, P_{1}\left(X_{1}\right), P_{1}\left(X_{0}\right)\right\} .
\end{gathered}
$$

Сосчитаем число перемен знака в каждой из них, применяя следующее

ПРАВИЛО ЗАМЕНЫ НУЛЕЙ. Нули в последовательностях заменяются положительными или отрицательными числами так, чтобы число перемен знака стало максимально возможным.

Пусть $\Pi_{1}, \Pi_{2}$ и $\Pi_{1}^{\prime}$ - сосчитанные по этому правилу числа перемен знака в последовательностях (106), а $K_{1}$ и $K_{2}$ - суммы кратностей корней $P_{1}(x)$ и $P_{2}(s)$, причем в число корней $P_{1}(x)$ включается корень $X_{0}=+\infty$ кратности 1 . Тогда верна

ЛЕмма 10. Выполняется иепочка неравенств

$$
\Pi_{1} \stackrel{(\mathrm{I})}{\leqslant} K_{1} \stackrel{(\mathrm{II})}{\leqslant} \Pi_{2} \stackrel{\text { (III) }}{\leqslant} K_{2} \stackrel{(\mathrm{IV})}{\leqslant} \Pi_{1}^{\prime}
$$

причем не может случиться, чтобы все они превратились в равенства.

Неравенство (105) является следствием леммы 10. Действительно, числа $P_{1}\left(X_{Q}\right), \ldots, P_{1}\left(X_{q+1}\right)$ положительнь ${ }^{\langle 37\rangle}$. Значит, если разность $t^{*}-t$ тоже положительна, то $\Pi_{1}=\Pi_{1}^{\prime}$ и все неравенства (107) преврашаются в равенства.

Итак, остается привести

ДоКАЗАТЕЛЬСтво ЛЕммы 10. Начнем с проверки неравенств (II) и (IV). Оба следуют из теоремы Гантмахера-Крейна (используемой также в ${ }^{\langle 16\rangle},\langle 22\rangle$ и в других комментариях).

По этой теореме сумма $K_{1}$ кратностей корней

$$
P_{1}(x)=\sum_{j=1}^{N} A_{j} C\left(S_{j}, x\right)
$$


не больше числа П перемен знака в последовательности коэффициентов $\left\{A_{j}\right\}$. Поэтому для проверки (II) достаточно сравнить последовательности

$$
\left\{A_{N}, \ldots, A_{1}\right\} \text { и }\left\{P_{2}\left(S_{N}\right), \ldots, P_{2}\left(S_{1}\right)\right\}
$$

и установить ${ }^{\langle 38\rangle}$, что $\Pi \leqslant \Pi_{2}$. Отсюда $K_{1} \leqslant \Pi \leqslant \Pi_{2}$.

Неравенство (IV) доказывается аналогично. Сумма $K_{2}$ кратностей корней

$$
P_{2}(s)=\sum_{k=0}^{Q} B_{k} C\left(s, X_{k}\right)+\left(t^{*}-t\right) I(s),
$$

где

$$
I(s)=\frac{2}{\pi\left(t^{*}-t\right)} \int_{t}^{t^{*}} C(s, x) d \sqrt{x},
$$

не больше числа П' перемен знака в последовательности коэффициентов

$$
\left\{B_{Q}, \ldots, B_{q+1}, t^{*}-t, B_{q}, \ldots, B_{1}, B_{0}\right\} .
$$

ЗАмечание. Коэффициент $t^{*}-t$ располагается между $B_{q+1}$ и $B_{q}$, так как согласно (103) $t$ и $t^{*}$ лежат между $X_{q+1}$ и $X_{q}$.

Сравним (108) с последовательностью

$$
\left\{P_{1}\left(X_{Q}\right), \ldots, P_{1}\left(X_{q+1}\right), t^{*}-t, P_{1}\left(X_{q}\right), \ldots, P_{1}\left(X_{1}\right), P_{1}\left(X_{0}\right)\right\}
$$

из (106). Так же, как в ${ }^{\langle 38\rangle}$, проверяется, что $\Pi^{\prime} \leqslant \Pi_{1}^{\prime\langle 39\rangle}$. Отсюда

$$
K_{2} \leqslant \Pi^{\prime} \leqslant \Pi_{1}^{\prime}
$$

Проверим теперь неравенство (III). Каждая перемена знака в последовательности $\left\{P_{2}\left(S_{N}\right), \ldots, P_{2}\left(S_{1}\right)\right\}$ дает (по теореме о промежуточном значении) нуль $P_{2}(s)$, а любой нуль в этой последовательности является кратным нулем $P_{2}(s)^{\langle 40\rangle}$. Поэтому число П 2 перемен знака (сосчитанное с применением правила замены нулей) не превосходит $K_{2}$.

Неравенство (I) доказывается аналогично ${ }^{\langle 41\rangle}$.

Чтобы доказать, что неравенства (III) и (IV) в (107) не могут оба превратиться в равенства, используем следующее

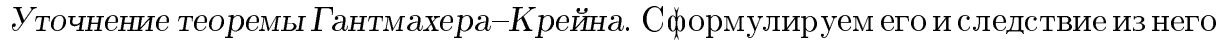
применительно к неравенствам (III) и (IV).

А) Пусть (см. (IV) и (109)) сумма $K_{2}$ кратностей корней

$$
P_{2}(s)=\sum_{k=0}^{Q} B_{k} C\left(s, X_{k}\right)+\left(t^{*}-t\right) I(s)
$$

равна числу П' перемен знакав последовательности (108). Тогда при малых $s>0$ (левее минимального корня $P_{2}(s)$ ) знак $P_{2}(s)$ совпадает со знаком $B_{Q}$. Следовательно, с учетом (103), $P_{2}(s)>0$ при этих $s$. 
В) Пусть (см. (III)) сумма $K_{2}$ кратностей корней $P_{2}(s)$ равна числу $\Pi_{2}$ перемен знака в последовательности

$$
\left\{P_{2}\left(S_{N}\right), \ldots, P_{2}\left(S_{1}\right)\right\}
$$

Тогда левее $S_{N}$ нет ни одного корня $P_{2}(s)$. Поэтому, если бы оба неравенства и (IV), и (III) - превратились в равенства, то $P_{2}\left(S_{N}\right)$ оказалось бы неотрицательным.

Но, учитывая (104), $S_{N}=s_{n\left(t^{*}\right)}\left(t^{*}\right)$ и (ввиду $\left.{ }^{\langle 38\rangle}\right) P_{2}\left(S_{N}\right)<0$. Значит, неравенства (107) не могут все сразу превратиться в равенства. Доказательство леммы 10 и теоремы 9 завершено $\langle 42\rangle$.

Исключительный случай. В этом случае верна $\langle 43\rangle$

TEOPEMA 10. Ecлu $\widehat{n}=1$, m.e. $d \hat{a}=d \hat{a}(s)=\delta(s-\widehat{s}) d s, m o$

$$
\max h(d a, t) \equiv h(d \hat{a}, t), \text { и, значит, } n(t) \equiv 1 \text { при всех } t \geqslant 0 .
$$

Если $\widehat{n}>1$, то для некоторого $t^{0} \in\left[0, \frac{3}{5}\right)$

$$
\max h(d a, t)=h(d \hat{a}, t) \text { nри всех } t, \quad 0 \leqslant t \leqslant t^{0},
$$

так что $n(t) \equiv \widehat{n}$ при $t \leqslant t^{0} ;$ при любом $t>t^{0}$ (как и в основном случае) максимум $h(d a, t)$ достигается не на мере dâ:

$$
\max h(d a, t) \neq h(d \hat{a}, t)
$$

Монотонность $n(t)$ при $t>t^{0}$ проверяется так же, как и в основном случае, а поскольку (см. (98)) $\max h(d a, t)$ достигается на мерах $d a$, сосредоточенных не более, чем в $\widehat{n}$ точках, функиия $n(t)$ не возрастает на всем отрезке $[0,1]$.

\section{$\S 12$. Комментарии}

1. Рассмотрим кривую $x_{0}=1, x_{1}=s, x_{2}=s^{2}, 0 \leqslant s \leqslant 1$, т.е. попросту дугу $\mathscr{D}$ параболы $x_{2}=x_{1}^{2}, 0 \leqslant x_{1} \leqslant 1$, лежащую в плоскости $x_{0}=1$. Ее замкнутую вьпуклую оболочку обозначим через $\mathscr{R}(\mathscr{D})$ (рис. 6 ). По теореме Рисса $[8$, с. 30] $\mathscr{R}(\mathscr{D})$ совпадает с множеством центров масс, расположенных на дуге $\mathscr{D}$. Иначе говоря, $\mathscr{R}(\mathscr{D})$ - это множество точек

$$
\left(x_{1}, x_{2}\right)=\left(I_{1}, I_{2}\right), \quad I_{1}=\int_{0}^{1} s d a(s), \quad I_{2}=\int_{0}^{1} s^{2} d a(s),
$$

где интегралы $I_{1,2}$ берутся по таким мерам Стилтьеса $d a(s) \geqslant 0$ на $[0,1]$, для которых $\int_{0}^{1} d a(s)=1$. Нам нужно найти максимум и минимум интеграла $I_{2}$ по всем таким мерам при дополнительном условии $I_{1}=\frac{1}{2}$. Это условие выделяет 


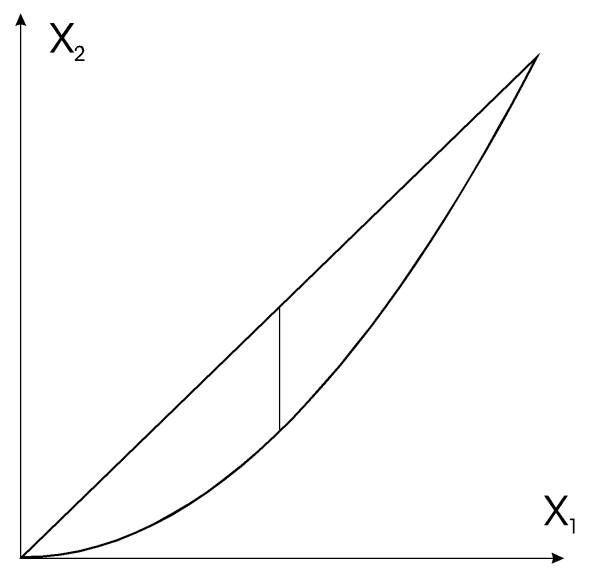

Рис. 6

из $\mathscr{R}(\mathscr{D})$ отрезок, в концах которого и достигаются искомые экстремумы. Итак, максимум и минимум интеграла $I_{2}$ достигаются на дискретных мерах

$$
d a_{1}(s)=\frac{\delta(s)+\delta(s-1)}{2} d s, \quad d a_{2}(s)=\delta\left(s-\frac{1}{2}\right) d s
$$

так что

$$
\max I_{2}=\int_{0}^{1} s^{2} d a_{1}(s)=\frac{1}{2}, \quad \min I_{2}=\int_{0}^{1} s^{2} d a_{2}(s)=\frac{1}{4}
$$

2. Квадратурами Гаусса для вычисления интеграла $\int_{-1}^{1} f(t) d t$ называются формулы вида $A(f)=\sum_{j=1}^{n} V_{j} f\left(\Lambda_{j}\right)$ с таким выбором весов $V_{j}$ и узлов $\Lambda_{j}$, чтобы (при заданном $n$ ) для многочленов भ( $(t)$ возможнно более высокой степени получались точные равенства

$$
\int_{-1}^{1} \mathscr{P}(t) d t=\sum_{j=1}^{n} V_{j} \mathscr{P}\left(\Lambda_{j}\right)
$$

В квадратурных формулах Гаусса в качестве узлов $\Lambda_{1}, \ldots, \Lambda_{n}$ берутся корни полинома Лежандра $L_{n}(t), t \in(-1,1),-$ тогда сушествуют и единственны такие веса $V_{1}, \ldots, V_{n}$, что точно интегрируются все многочлень $\mathscr{P}(t)$ до $(2 n-1)$ й степени.

При доказательстве теорем об универсальной последовательности перед нами возникнет

ЗАДАЧА. Найти такие узльи $\zeta_{1}, \ldots, \zeta_{n}$ и веса $c_{1}, \ldots, c_{n}$, чтобъ для любого многочлена $\mathscr{P}(t)$ степени $k \leqslant 2 n-1$ и для функиии $\nu(t)=t^{3 / 2} / 3$ выполнялись равенства

$$
\int_{0}^{1} \mathscr{P}(t) d \nu(t)=\sum_{j=1}^{n} c_{j} \mathscr{P}\left(\zeta_{j}\right)
$$


Мы увидим, что решение этой задачи существует и единственно, причем $\zeta_{1}, \ldots, \zeta_{n}$ - это квадраты ненулевых корней полинома Лежандра $L_{2 n+1}(x)$, а $c_{1}, \ldots, c_{n}$ определяются из системы линейных уравнений

$$
\sum_{j=1}^{n} c_{j} \zeta_{j}^{k}=\frac{1}{2 k+3}, \quad 0 \leqslant k \leqslant n-1 .
$$

3. Фактически задача была сделана при более сильных ограничениях.

4. Упрощение состоит в том, что сейсмические лучи с общим началом $A$ и общим концом $B$ ( $A$ и $B$ - точки на окружности), совершившие разное число оборотов вокруг центра круга, при переходек задаче в полуплоскости, отображаются в лучи с разныцми концами.

5 . Если в точке $(x, t)$ годограф $\Gamma$ имеет касательную с тангенсом угла наклона $p$, то $2 X(p)=x, 2 T(p)=t$.

В $[14$, c. 44$]$ приводится пример двухточечного годограффа $\Gamma$, по которому $X(p)$ и $T(p)$ определяются неоднозначно.

6. Лучи, начинаюшиеся в точке $A \in i$ и выходящие из $A$ под малым углом к оси $x$, не выходят из полосы $i$. Механический аналог - траектория шарика в желобе.

7. Функции $u_{1}(y)$ и $u_{2}(y), y \in i$, равноизмеримьи, если

$$
\operatorname{mes}\left\{y \in i, u_{1}(y)<t\right\}=\operatorname{mes}\left\{y \in i, u_{2}(y)<t\right\} \text { при всех } t .
$$

8. В [11] дается образное описание не изменяющего $Г$ преобразования $u(y)$ : говорится, что слои в волноводе можно тасовать.

9. Монотонная функция $\tau(p)$ дифференцируема почти всюду и на множестве полной меры $\tau^{\prime}(p)=-X(p)$. Можно доказать, что $\tau(p)$ абсолютно непрерывна при $p \in(0,1)$ и имеет скачок при $p=1$, так что

$$
\tau(p)=\tau(1-0)+\int_{p}^{1} X(q) d q .
$$

10. Формула (12) - это иначе записанная формула (8).

11. Основные, не поддававшиеся старым методам и остаюшиеся пока нерешенными проблемы в классической задаче обрашения годографа - это построение нижней границы полосы $G$ (в случае, когда волновод один) и аналогичное более общее построение в случае двух или нескольких волноводов.

12. В [5] предлагался более сложный алгоритм:

а) Рассмотрим бесконечную вправо и вниз матрицу

$$
M(t)=\left(\begin{array}{ccccc}
1 & t & t^{2} & t^{3} & \ldots \\
1 / 3 & 1 / 5 & 1 / 7 & 1 / 9 & \ldots \\
1 / 5 & 1 / 7 & 1 / 9 & 1 / 11 & \ldots \\
1 / 7 & 1 / 9 & 1 / 11 & 1 / 13 & \ldots \\
\ldots \ldots \ldots \ldots \ldots \ldots \ldots \ldots
\end{array}\right)
$$

и ее части $M_{k}(t), k \geqslant 1$, содерэсащие $k+1$ строк и $k+1$ столбцов:

$$
M_{1}(t)=\left(\begin{array}{cc}
1 & t \\
1 / 3 & 1 / 5
\end{array}\right), \quad M_{2}(t)=\left(\begin{array}{ccc}
1 & t & t^{2} \\
1 / 3 & 1 / 5 & 1 / 7 \\
1 / 5 & 1 / 7 & 1 / 9
\end{array}\right), \ldots
$$


б) Обозначим через $t_{k}$ наименьиий корень уравнения $\operatorname{det} M_{k}(t)=0$; отметим, что $t_{k} \in\left(0, t_{k-1}\right)$, это облегчает вычисление $t_{k}$.

в) Пологим $U_{k}=1 / \sqrt{1-t_{k}}$.

13. Известно, что при $k \rightarrow \infty$ наименьший положительньй корень $\lambda_{k}$ многочлена Лежандра $L_{2 k+1}(x)$ убывает как $O(1 / k)$ :

$$
\lambda_{k}=O\left(\frac{1}{k}\right)
$$

Поэтому для универсальной последовательности

$$
U_{k}=\left(1-\lambda_{k}^{2}\right)^{-1 / 2}, \quad k=1,2,3, \ldots
$$

вьполняется оценка $U_{k}-1=O\left(1 / k^{2}\right)$. Приведем простое доказательство (110). Согласно [16, часть 2 , отдел VI, задача 93 , с. 104,311$] L_{2 k+1}(\cos x)$ является тригонометрическим многочленом вида

$$
c_{0} \cos x+c_{1} \cos 3 x+\cdots+c_{k} \cos (2 k+1) x
$$

с коэффициентами, вычисляемыми по формулам

$$
c_{0}=2 g_{k} g_{k+1}, \quad c_{1}=2 g_{k-1} g_{k+2}, \quad \ldots, \quad c_{k-1}=2 g_{1} g_{2 k}, \quad c_{k}=2 g_{0} g_{2 k+1},
$$

где

$$
g_{0}=1, \quad g_{k}=\frac{1 \cdot 3 \cdots(2 k-1)}{2 \cdot 4 \cdots 2 k}, \quad k=1,2,3, \ldots
$$

Значит, коэффициенты в (111) положстельны и возрастают:

$$
0<c_{0}<c_{1}<\cdots<c_{k}
$$

Рассмотрим множество тригонометрических многочленов вида (111), удовлетворяющих условию (112). Из принадлежности $L_{2 k+1}(\cos x)$ этому множеству следует (см. [17]), что расстояния между соседними корнями $L_{2 k+1}(\cos x)$ убывают с ростом $k$ как $O(1 / k)$, т.е. следует оценка (110).

С помощью приема, предложенного в [17], можно оценить $\lambda_{k}$ и более точно:

$$
\sin \alpha_{k}<\lambda_{k}<\sin \beta_{k}, \quad \text { где } \quad \alpha_{k}=\frac{\pi}{4 k}, \quad \beta_{k}=\frac{3 \pi}{4(k+1)}, \quad k \geqslant 1 \text {; }
$$

для $U_{k}$ отсюда следует, что $1 /\left(\cos \alpha_{k}\right)<U_{k}<1 /\left(\cos \beta_{k}\right), k \geqslant 1$, а значит,

$$
1+\frac{0.3}{k^{2}}<U_{k}<1+\frac{2.8}{k^{2}}, \quad k \geqslant 1
$$

14. Рекуррентные формулы для полиномов Лежандра

$$
L_{0}(x)=1, \quad L_{1}(x)=x, \quad L_{n}(x)=\left(2-\frac{1}{n}\right) x L_{n-1}(x)-\left(1-\frac{1}{n}\right) L_{n-2}(x)
$$


показывают, что $L_{n}(x)$ содержит степени $x$ только той же четности, какова четность $n$. Поэтому функция $P_{n}(t)$, определяемая равенством

$$
x P_{n}\left(x^{2}\right)=L_{2 n+1}(x),
$$

- полином степени $n$. Так как $L_{2 n+1}(x) / x$ имеет $n$ пар корней $\pm \alpha_{j}, 1 \leqslant j \leqslant n$, на $[-1,1]$, то $P_{n}(t)$ имеет $n$ корней $\alpha_{j}^{2}, 1 \leqslant j \leqslant n$, на $[0,1]$.

Как известно, полином Лежандра $L_{n}(x)$ степени $n$ ортогонален на $[-1,1]$ всем полиномам степени $k<n$ :

$$
\int_{-1}^{1} x^{k} L_{n}(x) d x=0, \quad 0 \leqslant k \leqslant n-1
$$

Поэтому

$$
\int_{0}^{1} x^{2 k+1} L_{2 n+1}(x) d x=0, \quad 0 \leqslant k \leqslant n-1 .
$$

Отсюда (ввиду равенства $\left.d \nu(t)=\frac{1}{2} t^{1 / 2} d t\right)$ при $0 \leqslant k \leqslant n-1$

$$
\begin{aligned}
\int_{0}^{1} t^{k} P_{n}(t) d \nu(t) & =\frac{1}{2} \int_{0}^{1} t^{k+1 / 2} P_{n}(t) d t \\
& =\frac{1}{2} \int_{0}^{1} x^{2 k+1} P_{n}\left(x^{2}\right) d\left(x^{2}\right) \\
& =\int_{0}^{1} x^{2 k+1} L_{2 n+1}(x) d x=0
\end{aligned}
$$

Любой многочлен $\mathscr{P}(t)$ степени $s \leqslant 2 n-1$ можно (разделив с остатком на $P_{n}(t)$ ) представить в виде

$$
\mathscr{P}(t)=\mathscr{A}(t)+\mathscr{B}(t), \quad \mathscr{A}(t)=\sum_{k=0}^{n-1} A_{k} t^{k} P_{n}(t), \quad \mathscr{B}(t)=\sum_{k=0}^{n-1} B_{k} t^{k}
$$

По доказанному

$$
\int_{0}^{1} \mathscr{A}(t) d \nu(t)=0
$$

Корни $\zeta_{j}=\alpha_{j}^{2}$ полинома $P_{n}(t)$ являются также корнями $\mathscr{A}(t)$. Поэтому

$$
\int_{0}^{1} \mathscr{A}(t) d \nu(t)=\sum_{j=1}^{n} c_{j} \mathscr{A}\left(\zeta_{j}\right)
$$

при любых $c_{1}, \ldots, c_{n}$, в частности, и при таких, которые определяются из системы линейных уравнений

$$
\sum_{j=1}^{n} c_{j} \zeta_{j}^{k}=\frac{1}{2 k+3}, \quad 0 \leqslant k \leqslant n-1
$$


Правые части этих уравнений - интегралы от $t^{k}$ по $d \nu(t)$ :

$$
\int_{0}^{1} t^{k} d \nu(t)=\frac{1}{2} \int_{0}^{1} t^{k+1 / 2} d t=\frac{1}{2 k+3}, \quad 0 \leqslant k \leqslant n-1
$$

Следовательно, для так определенных $c_{1}, \ldots, c_{n}$

$$
\int_{0}^{1} \mathscr{B}(t) d \nu(t)=\sum_{j=1}^{n} c_{j} \mathscr{B}\left(\zeta_{j}\right),
$$

а значит, и

$$
\int_{0}^{1} \mathscr{P}(t) d \nu(t)=\sum_{j=1}^{n} c_{j} \mathscr{P}\left(\zeta_{j}\right) .
$$

Из приведенного доказательства следует, что задачи 1 и 2 эквивалентны и их решение существует.

Остается доказать единственность найденного решения. Допустим, что сушествует еше одно решение $c_{j}^{*}, \zeta_{j}^{*}, 1 \leqslant j \leqslant n$. Объединим $\zeta_{j}$ и $\zeta_{j}^{*}, 1 \leqslant j \leqslant n$, и обозначим все попарно различные точки этого объединения через $\xi_{1}, \ldots, \xi_{q}$, где $q \leqslant 2 n$. Решая систему линейных уравнений

$$
\sum_{j=1}^{q} e_{j} \xi_{j}^{k}=\frac{1}{2 k+3}, \quad 0 \leqslant k \leqslant q-1
$$

получаем единственный набор чисел $e_{1}, \ldots, e_{q}$, удовлетворяющих (вместе с $\left.\xi_{1}, \ldots, \xi_{q}\right)$ этой системе. Однако $e_{1}, \ldots, e_{q}$, равные $c_{1}, \ldots, c_{n}$ в точках $\xi_{j}$, совпадающих с $\zeta_{1}, \ldots, \zeta_{n}$, и равные нулю в остальных точках $\xi_{j},-$ это один такой набор чисел, а $e_{1}, \ldots, e_{q}$, равные $c_{1}^{*}, \ldots, c_{n}^{*}$ в точках $\xi_{j}^{*}$, совпадаюших с $\zeta_{1}^{*}, \ldots, \zeta_{n}^{*}$, и равные нулю в остальных точках $\xi_{j}$, - это другой такой набор. Полученное противоречие доказывает теорему 8 .

15. Нетрудно проверить (действуя, как в $\S 7$ ), что при любом $m \geqslant 1$ и любом $t>0$ аппроксимация $r_{0}(s)$ функиии $\varphi(s)=\kappa(s, t)$ существует и однозначно ( с точностью до нумерации слагаемых) определяется условиями (48).

16. Разобьем доказательство леммы 2 на три части.

А) Пусть (см. (40)-(45)) точки наборов $\mathscr{S}$ и $\mathscr{S}^{*}$ чередуются:

$$
s_{1} \geqslant s_{1}^{*} \geqslant \cdots \geqslant s_{m} \geqslant s_{m}^{*} \geqslant s_{m+1}>s_{m+1}^{*}>0 .
$$

Докажем, что тогда полюсы $w_{k}$ и $w_{k}^{*}, 1 \leqslant k \leqslant m$, аппроксимаций $r(\mathscr{S}, s)$ и $r\left(\mathscr{S}^{*}, s\right)$ тоже чередуются, причем неравенства строгие:

$$
w_{1}>w_{1}^{*}>\cdots>w_{m}>w_{m}^{*}>w_{m+1}=w_{m+1}^{*}=0 .
$$

Согласно (42) и (45) при всех $j, 1 \leqslant j \leqslant m+1$,

$$
r\left(\mathscr{S}, s_{j}\right)=\varphi\left(s_{j}\right) \leqslant r\left(\mathscr{S}^{*}, s_{j}\right), \quad r\left(\mathscr{S}^{*}, s_{j}^{*}\right)=\varphi\left(s_{j}^{*}\right) \leqslant r\left(\mathscr{S}, s_{j}^{*}\right) .
$$


Поэтому для функции

$$
\rho(s)=r(\mathscr{S}, s)-r\left(\mathscr{S}^{*}, s\right)=d_{0}-d_{0}^{*}+\sum_{k=1}^{m+1}\left(\frac{d_{k}}{s+w_{k}}-\frac{d_{k}^{*}}{s+w_{k}^{*}}\right)
$$

сумма $K$ кратностей корней на полуоси $s>0$ и даже сумма $K^{\prime}$ кратностей корней на отрезке $\left[s_{m+1}^{*}, s_{1}\right]$ не меньше $2 m+1: K \geqslant K^{\prime} \geqslant 2 m+1$. Приведем в (115) подобные члены, упорядочим полюсы по убыванию и запишем (115) в виде

$$
\rho(s)=c_{0}+\sum_{k=1}^{p} \frac{c_{k}}{s+x_{k}}, \quad x_{1}>\cdots>x_{p}=0, \quad p \leqslant 2 m+1 .
$$

По теореме Гантмахера-Крейна (см. [8, с. 62], [18, с. 36-38] либо [19]) число П перемен знака в последовательности коэффичиентов $c_{0}, \ldots, c_{p}$ не менвие $K$. Так как $\Pi \leqslant p$, отсюда следует, что $p=\Pi=K=K^{\prime}=2 m+1$. Поэтому $\rho(s) \neq 0$ при $0<s<s_{m+1}^{*}$ и, поскольку $s_{m+1}^{*}<s_{m+1}$ (см. (113)), $\rho(s)>0$ на $\left(0, s_{m+1}^{*}\right]$. Значит, $c_{p}=c_{2 m+1}=d_{m+1}-d_{m+1}^{*}>0$ и (114) доказано: если полюсы $w_{k}$ и $w_{k}^{*}$ располагаются в любом другом порядке, то $\Pi<2 m+1$.

В) Взяв малое $\varepsilon>0$, рассмотрим последовательность наборов

$$
\mathscr{I}_{n}=\left\{s_{n, 1}, \ldots, s_{n, m+1}\right\}, \quad s_{n, 1}>\cdots>s_{n, m+1}>0, \quad n=1, \ldots, N,
$$

обладающую тремя свойствами: а) $\mathscr{S}_{1}=\mathscr{S}$, б) $s_{N, 1}<\varepsilon$, в) точки наборов $\mathscr{S}_{n}$ и $\mathscr{S}_{n+1}(1 \leqslant n<N)$ чередуются:

$$
s_{n, 1}>s_{n+1,1}>\cdots>s_{n, m+1}>s_{n+1, m+1}>0 .
$$

Согласно А) для аппроксимаций $r\left(\mathscr{S}_{n}, s\right)$ и $r\left(\mathscr{S}_{n+1}, s\right)$

$$
w_{n, 1}>w_{n+1,1}>\cdots>w_{n, m+1}=w_{n+1, m+1}=0 .
$$

Отсюда (cp. с (50))

$$
w_{1}=w_{1,1}>w_{N, 1}
$$

С) При $\varepsilon \rightarrow 0$ аппроксимация $r\left(\mathscr{S}_{N}, s\right)$, очевидно, стремится к аппроксимации $r_{0}(s)$ (см. $\left.(47),(48)\right)$. Отсюда ввиду (116) следует (50), что и доказывает лемму 2.

17. Докажем, что утверждение 1 следует из утверждения 3.

Ясно (см. определение $H(p)$ в начале $\S 5)$, что

$$
H(p)=\sup \Psi(p) \text { по всем ступенчатым } u(y) \in \mathscr{U} .
$$

Эквивалентное утверждение: пусть

$$
h(t)=H(\sqrt{1-t})=\sup h(d a, t) \text { по всем мерам (38), }
$$

тогда $h(t)$ совпадает с $\sup h(d a, t)$ по обвединению $\mathscr{A}_{n}, n \geqslant 1$. 
Значит, если положить $h_{k}(t)=\sup h(d a, t)$ по $\mathscr{A}_{k}$ и допустить, что (вопреки утверждению 1)) $h_{k}(t)$ строго меньше $h(t)$, хотя $t \geqslant t_{k}$, то найдутся такое $n$ и такая дискретная мера $d a^{0}$ из $\mathscr{A}_{n}$, что $h\left(d a^{0}, t\right)>h_{k}(t)$.

Найдем $\max h(d a, t)$ по всем $d a \prec d a^{0}$. С одной стороны, этот максимум не меньше $h\left(d a^{0}, t\right)$ и, значит, больше $h_{k}(t)$; следовательно, согласно [2] он достигается на некоторой мере $d a \in \mathscr{A}_{m+1}$, где $m \geqslant k$; с другой стороны, по утверждению 3 при $t \geqslant t_{k} \geqslant t_{m}$ вьполняется неравенство (51), и лемма 2 приводит нас к противоречию с леммой 1 .

18. При $\sum_{j=1}^{k} a_{j}=a<1$ добавим к мере $d \tilde{a} \in \widetilde{\mathscr{A}}_{k}$ слагаемое

$$
(\alpha+1-a) \delta(s-S) d s-\alpha \delta\left(s-s_{1}\right) d s
$$

выбрав $\alpha \in\left(0, a_{1}\right)$ столь малым, а $S$ столь большим, что

$$
\alpha \varphi\left(s_{1}\right)<\varepsilon, \quad \frac{\alpha+1-a}{S+x}<\frac{\alpha}{s_{1}+x} \text { при } x \in[0,1] .
$$

Тогда

$$
g(d a, x)=g(d \tilde{a}, x)-\frac{\alpha}{s_{1}+x}+\frac{\alpha+1-a}{S+x}<M(x) \text { на }[0,1],
$$

так что $d a=d \tilde{a}-\alpha \delta\left(s-s_{1}\right) d s+(\alpha+1-a) \delta(s-S) d s \in \mathscr{A}_{k+1}$, и при этом

$$
h(d a, t)=h(d \tilde{a}, t)-\alpha \varphi\left(s_{1}\right)+(\alpha+1-a) \varphi(S)>h(d \tilde{a}, t)-\varepsilon .
$$

19. В последовательности (56) мер $d a_{n}(s)=\sum_{j=1}^{k} a_{n j} \delta\left(s-s_{n j}\right) d s$ коэффициенты $a_{n j}$ неотрицательны и в сумме равны 1 , а следовательно, ограничены. Функция

$$
\varphi(s)=\kappa\left(s, t^{0}\right)=\frac{2}{\pi} \frac{\operatorname{arctg} \sqrt{s / t^{0}}}{\sqrt{s}} \rightarrow 0 \text { при } s \rightarrow+\infty .
$$

Значит, сумма

$$
\sum a_{n j} \varphi\left(s_{n j}\right) \text { по } j \in J_{\infty}
$$

сходится к нулю при $n \rightarrow+\infty$. То же верно и для суммы

$$
\sum a_{n j} \varphi\left(s_{n j}\right) \text { по } j \in J_{0},
$$

так как $\varphi(s)$ ограничена и (как нетрудно проверить)

$$
a_{n j} \rightarrow 0 \text { при } s_{n j} \rightarrow 0
$$

Докажем (117). Ширина волновода, соответствующего мере $d a_{n}$, равна $h_{n}=\sum_{j=1}^{k} a_{n j} \kappa\left(s_{n j}, 0\right)=\sum_{j=1}^{k} a_{n j} / \sqrt{s_{n j}}$. Числа $h_{n}$, очевидно, ограничены: $h_{n} \leqslant \inf T(p), 0<p<1$. Действительно, каждый сейсмический луч $L(p)$ с параметром $p, 0<p<1$, пересекает волновод насквозь. Значит, путь, который сейсмический импульс проходит в волноводе по нисходящей дуге луча $L(p)$, больше $h_{n}$ и не превосходит $T(p)$ (так как скорость импульса в волноводе не больше 1 ). Поэтому $a_{n j}=O\left(\sqrt{s_{n j}}\right)$ при $s_{n j} \rightarrow 0$. 
Итак, $\sum a_{n j} \varphi\left(s_{n j}\right)$ по $j \in J_{\infty} \cup J_{0}$ сходится к нулю при $n \rightarrow+\infty$, и, значит, $\sum a_{n j} \varphi\left(s_{n j}\right)$ по $j \in J$ сходится к $h_{k}\left(t^{0}\right)$, т.е. $h\left(d a^{0}, t^{0}\right)=h_{k}\left(t^{0}\right)$.

20. При $s_{1}^{0} \geqslant 1$ возьмем $0<\varepsilon \leqslant(1-a) / a_{1}^{0}$, положим

$$
k=1+\varepsilon, \quad a_{1}=k a_{1}^{0}, \quad s_{1}=k s_{1}^{0}+\varepsilon
$$

и заменим $\left\{a_{1}^{0}, s_{1}^{0}\right\}$ в $d a^{0}$ на $\left\{a_{1}, s_{1}\right\}$ в $d a$, не меняя остальные $a_{j}^{0}, s_{j}^{0}, 2 \leqslant j \leqslant m$.

Тогда

а) сумма коэффициентов в $d a$ равна $a+\varepsilon a_{1}^{0} \leqslant 1$;

б) $d a \prec d a^{0}$, так как

$$
g\left(d a^{0}, x\right)-g(d a, x)=\frac{a_{1}^{0}}{s_{1}^{0}+x}-\frac{k a_{1}^{0}}{k s_{1}^{0}+\varepsilon+x}>0 \text { на }[0,1),
$$

поскольку с точностью до положительного множителя эта разность равна

$$
k s_{1}^{0}+\varepsilon+x-k\left(s_{1}^{0}+x\right)=\varepsilon+x(1-k)=\varepsilon(1-x) ;
$$

в) $h\left(d a, t^{0}\right)-h\left(d a^{0}, t^{0}\right)=a_{1} \varphi\left(s_{1}\right)-a_{1}^{0} \varphi\left(s_{1}^{0}\right)$, где

$$
\varphi(s)=\frac{2}{\pi} \frac{\operatorname{arctg} \sqrt{s / t^{0}}}{\sqrt{s}},
$$

так что (ввиду неравенства $s_{1}^{0} \geqslant 1$ ) вьполняется (61).

Действительно, $k^{2}=(1+\varepsilon)^{2}>1+2 \varepsilon=k+\varepsilon>k+\varepsilon / s_{1}^{0}$. Значит,

$$
k>\sqrt{\frac{k s_{1}^{0}+\varepsilon}{s_{1}^{0}}} .
$$

Отсюда (с учетом равенств $a_{1}=k a_{1}^{0}, s_{1}=k s_{1}^{0}+\varepsilon$ )

$$
a_{1} \varphi\left(s_{1}\right)-a_{1}^{0} \varphi\left(s_{1}^{0}\right)>\frac{2 a_{1}^{0}}{\pi} \operatorname{arctg} \sqrt{\frac{s_{1}^{0}}{t^{0}}}\left(\frac{k}{\sqrt{k s_{1}^{0}+\varepsilon}}-\frac{1}{\sqrt{s_{1}^{0}}}\right)>0 .
$$

21. Доказано даже больше, а именно, что при $d a=d a^{0}$ функиионал $h\left(d a, t^{0}\right)$

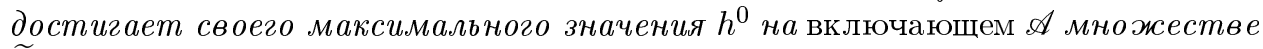
$\widetilde{\mathscr{A}}$ мер $d a \geqslant 0$, для которых

$$
\int_{0}^{\infty} d a \leqslant 1, \quad g(d a, x)=\int_{0}^{\infty} \frac{d a(s)}{s+x} \leqslant M(x) \text { nрu } x \in[0,1] .
$$

22. Пусть для определенности $t^{0}<x_{p}<\cdots<x_{1}$. Положим

$$
d \theta(x)=\frac{2}{\pi} d \sqrt{x}
$$

тогда

$$
\varphi(s)=\int_{t^{0}}^{\infty} \frac{d \theta(x)}{s+x}=\varphi_{0}(s)+\varphi_{1}(s)+\cdots+\varphi_{p}(s)
$$


где

$$
\varphi_{p}(s)=\int_{t^{0}}^{x_{p}} \frac{d \theta(x)}{s+x}, \ldots, \quad \varphi_{1}(s)=\int_{x_{2}}^{x_{1}} \frac{d \theta(x)}{s+x}, \quad \varphi_{0}(s)=\int_{x_{1}}^{\infty} \frac{d \theta(x)}{s+x}
$$

По теореме Гантмахера-Крейна (cp. $\left.{ }^{\langle 16\rangle}\right)$ сумма кратностей корней $r(s)-\varphi(s)$ не больше числа П перемен знака в разложении

$$
r(s)-\varphi(s)=d_{0}-\varphi_{0}(s)+\frac{d_{1}}{s+x_{1}}-\varphi_{1}(s)+\cdots+\frac{d_{p}}{s+x_{p}}-\varphi_{p}(s) .
$$

Так как $\Pi \leqslant 2 p+1\left(\Pi=2 p+1\right.$, если все $\left.d_{k}>0,0 \leqslant k \leqslant p\right)$ и так как все корни $r(s)-\varphi(s)$ минимум двукратные (поскольку $r \geqslant \varphi$ ), то число $q$ корней $r(s)-\varphi(s)$ не больше $p$. По условию $r(s)-\varphi(s)$ имеет не менее $m$ корней. Значит, $m \leqslant q \leqslant p$.

Если среди коэффициентов $d_{1}, \ldots, d_{p}$ лишь $n$ положительных, то число П перемен знака в разложении $(118)$ не больше $2 n+1$. Снова применяя теорему Гантмахера-Крейна, получаем $n \geqslant q$.

23. Коэффициенты $a_{j}^{0}$ при $m<j \leqslant q$ (которые до сих пор не были определены) считаются равными нулю.

24. Выпишем определитель $\Delta_{m}$ системы (73). При $m=2$

$$
\Delta_{2}=\left|\begin{array}{cccc}
1 & 1 /\left(s_{1}^{0}+x_{1}\right) & 1 /\left(s_{1}^{0}+x_{2}\right) & 1 /\left(s_{1}^{0}+x_{3}\right) \\
1 & 1 /\left(s_{2}^{0}+x_{1}\right) & 1 /\left(s_{2}^{0}+x_{2}\right) & 1 /\left(s_{2}^{0}+x_{3}\right) \\
0 & -1 /\left(s_{1}^{0}+x_{1}\right)^{2} & -1 /\left(s_{1}^{0}+x_{2}\right)^{2} & -1 /\left(s_{1}^{0}+x_{3}\right)^{2} \\
0 & -1 /\left(s_{2}^{0}+x_{1}\right)^{2} & -1 /\left(s_{2}^{0}+x_{2}\right)^{2} & -1 /\left(s_{2}^{0}+x_{3}\right)^{2}
\end{array}\right|
$$

в общем случае формулы для $\Delta_{m}$ аналогичны.

Так как ядро Коши $C(s, x)=1 /(s+x)$ является обобшенным вполне положительным ядром (ЕТР-ядром) [18] и остается ЕТР-ядром, если доопределить его при $x=\infty$, положив $C(s, \infty)=1$ для всех $s>0[3]$, то $\Delta_{m} \neq 0$. Отметим, что, используя $[3$, с. 23,24$]$, нетрудно доказать, что $\Delta_{m} \neq 0$, вычислив $\left|\Delta_{m}\right|$ в явном виде

$$
\left|\Delta_{m}\right|=\prod_{1 \leqslant j<k \leqslant m}\left(s_{j}-s_{k}\right)^{4} \prod_{1 \leqslant j<k<2 m}\left(x_{j}-x_{k}\right) / \prod_{\substack{1 \leqslant j \leqslant m, 1 \leqslant k<2 m}}\left(s_{j}+x_{k}\right)^{2}
$$

25. Легко выписать якобиан $J_{m}$ перехода от координат $\left\{a_{j}, s_{j}\right\}, j=1, \ldots, m$, к координатам (78). Например, при $m=2$ это - определитель

$$
J_{2}=\left|\begin{array}{cccc}
1 & 1 & 0 & 0 \\
1 /\left(s_{1}+x_{1}\right) & 1 /\left(s_{2}+x_{1}\right) & -a_{1} /\left(s_{1}+x_{1}\right)^{2} & -a_{2} /\left(s_{2}+x_{1}\right)^{2} \\
1 /\left(s_{1}+x_{2}\right) & 1 /\left(s_{2}+x_{2}\right) & -a_{1} /\left(s_{1}+x_{2}\right)^{2} & -a_{2} /\left(s_{2}+x_{2}\right)^{2} \\
1 /\left(s_{1}+x_{3}\right) & 1 /\left(s_{2}+x_{3}\right) & -a_{1} /\left(s_{1}+x_{3}\right)^{2} & -a_{2} /\left(s_{2}+x_{3}\right)^{2}
\end{array}\right|
$$

В обшем случае формулы для $J_{m}$ аналогичны, так что $J_{m} \neq 0$ : с точностью до замены $s_{j}$ на $s_{j}^{0}\left(\right.$ см. $\left.^{\langle 24\rangle}\right)$

$$
J_{m}=a_{1} \cdots a_{m} \Delta_{m}
$$


26. По построению (см. (80)) разность $g\left(\mathscr{N}^{0}, x\right)-g\left(\mathscr{N}^{*}(\lambda), x\right)$ положительна при любом $x=x_{k}$ и равна $\lambda \xi_{k}, k=1, \ldots, p$.

При достаточно большом $C>0$ для производной этой разности по $x$ на всем отрезке $[0,1]$, с учетом ${ }^{\langle 25\rangle}$, выполняется неравенство

$$
\left|g^{\prime}\left(\mathscr{N}^{0}, x\right)-g^{\prime}\left(\mathscr{N}^{*}(\lambda), x\right)\right| \leqslant \sum_{j=1}^{m}\left|\frac{a_{j}^{*}}{\left(s_{j}^{*}+x\right)^{2}}-\frac{a_{j}^{0}}{\left(s_{j}^{0}+x\right)^{2}}\right| \leqslant C \lambda .
$$

Значит, если в (83) положить $\varepsilon=\min \left(\xi_{1} / C, \ldots, \xi_{p} / C\right)$, то

$$
g\left(\mathscr{N}^{0}, x\right)-g\left(\mathscr{N}^{*}(\lambda), x\right)>0
$$

при всех $x \in V$, что и требовалось доказать.

27. Согласно $\langle 26\rangle$

$$
g\left(\mathscr{N}^{*}(\lambda), x\right)<g\left(\mathscr{N}^{0}, x\right) \leqslant M(x) \text { при } x \in V .
$$

Дополнение к $V$ пересечем с отрезком $[0,1]$ и полученное замкнутое множество обозначим через $F$. Докажем, что на $F$

$$
g\left(\mathscr{N}^{*}(\lambda), x\right)<M(x)
$$

если $\lambda$ достаточно мало.

Мажоранта $M(x)$, а значит, и разность

$$
\rho(x)=M(x)-g\left(\mathscr{N}^{0}, x\right)
$$

полунепрерывны снизу в любой точке отрезка [0, 1]. Поэтому [20, с. 103] $\rho(x)$ достигает минимума на замкнутом множестве $F$ в некоторой точке $x_{0} \in F$ :

$$
\rho_{0}=\rho\left(x_{0}\right)=\min \rho(x) \text { по всем } x \in F,
$$

причем $\rho_{0}>0$, так как $\rho(x)>0$ на $F$. Остается взять $\lambda$ столь мальм, что $g\left(\mathscr{N}^{*}(\lambda), x\right)-g\left(\mathscr{N}^{0}, x\right)<\rho_{0}$ при всех $x \in[0,1]$. Тогда

$$
g\left(\mathscr{N}^{*}(\lambda), x\right)<g\left(\mathscr{N}^{0}, x\right)+\rho_{0} \leqslant M(x) \text { при всех } x \in F,
$$

т.е. $g\left(\mathscr{N}^{*}(\lambda), x\right)<M(x)$ при всех $x \in[0,1]$.

28. Так как (см. (73)) $\varphi\left(s_{j}^{0}\right)=r\left(s_{j}^{0}\right), 1 \leqslant j \leqslant m$, то

$$
h\left(\mathscr{N}^{0}\right)=\int_{0}^{\infty} \varphi(s) d a^{0}(s)=\int_{0}^{\infty} r(s) d a^{0}(s) .
$$

Отсюда (ср. с (67))

$$
h\left(\mathscr{N}^{0}\right)=d_{0}+\sum_{k=1}^{2 m-1} d_{k} g\left(\mathscr{N}^{0}, x_{k}\right)
$$


В сумме

$$
h(\mathscr{N})=\int_{0}^{\infty} r(s) d a(s)+\int_{0}^{\infty}(\varphi(s)-r(s)) d a(s)
$$

первое слагаемое (ср. с (68)) равно $d_{0}+\sum_{k=1}^{2 m-1} d_{k} g\left(\mathscr{N}, x_{k}\right)$. Значит, ввиду (79), $(80)$ и (81) на луче (82) оно равно

$$
h\left(\mathscr{N}^{0}\right)+\sum_{k=1}^{2 m-1} d_{k}\left(g\left(\mathscr{N}^{*}, x_{k}\right)-g\left(\mathscr{N}^{0}, x_{k}\right)\right)=h\left(\mathscr{N}^{0}\right)-\lambda \sum_{k=1}^{p} d_{k} \xi_{k}=h\left(\mathscr{N}^{0}\right)+\lambda .
$$

Оценим второе слагаемое в (119). Так как $d a(s)=\sum_{j=1}^{m} a_{j} \delta\left(s-s_{j}\right) d s$, то

$$
\int_{0}^{\infty}(\varphi(s)-r(s)) d a(s)=\sum_{j=1}^{m} a_{j}\left(\varphi\left(s_{j}\right)-r\left(s_{j}\right)\right) .
$$

Согласно $(73) \varphi\left(s_{j}\right)-r\left(s_{j}\right)=o\left(s_{j}-s_{j}^{0}\right)$. Значит, с учетом $\langle 25\rangle$, на луче (82) второе слагаемое в (119) равно $o(\lambda)$. Итак, $h\left(\mathscr{N}^{*}(\lambda)\right)-h\left(\mathscr{N}^{0}\right)=\lambda+o(\lambda)>0$.

29. Докажем, что в множестве (85) менее $2 m$ точек.

В соответствии с неравенствами $0<s_{m}^{0}<\cdots<s_{1}^{0}$ представим $g\left(d a^{1}, x\right)$ в виде суммы $2 m+1$ неотрицательных слагаемых:

$$
g\left(d a^{1}, x\right)=\int_{0}^{\infty} \frac{d a^{1}(s)}{s+x}=g_{0}(x)+g_{1}(x)+\cdots+g_{m}(x)+\sum_{j=1}^{m} \frac{a_{j}}{s_{j}^{0}+x},
$$

положив

$$
\begin{gathered}
g_{m}(x)=\int_{0}^{s_{m}^{0}-0} \frac{d a^{1}(s)}{s+x}, \ldots, \quad g_{1}(x)=\int_{s_{2}^{0}+0}^{s_{1}^{0}-0} \frac{d a^{1}(s)}{s+x}, \quad g_{0}(x)=\int_{s_{1}^{0}+0}^{\infty} \frac{d a^{1}(s)}{s+x}, \\
\frac{a_{j}}{s_{j}^{0}+x}=\int_{s_{j}^{0}-0}^{s_{j}^{0}+0} \frac{d a^{1}(s)}{s+x}, \quad 1 \leqslant j \leqslant m .
\end{gathered}
$$

Разность

$$
g\left(d a^{1}, x\right)-g\left(d a^{0}, x\right)=\int_{0}^{\infty} \frac{d a^{1}(s)}{s+x}-\sum_{j=1}^{m} \frac{a_{j}^{0}}{s_{j}^{0}+x}
$$

запишем в виде

$$
g_{0}(x)-\frac{a_{1}^{0}-a_{1}}{s_{1}^{0}+x}+g_{1}(x)-\frac{a_{2}^{0}-a_{2}}{s_{2}^{0}+x}+\cdots+g_{m-1}(x)-\frac{a_{m}^{0}-a_{m}}{s_{m}^{0}+x}+g_{m}(x) .
$$

Пусть П - число перемен знака в этом разложении. Чтобы получить нужную нам оценку для числа корней $g\left(d a^{1}, x\right)-g\left(d a^{0}, x\right)$, доопределим ядро Коши $C(s, x)=1 /(s+x)$ при $x=\infty$, положив $C(s, \infty)=1$ (ср. с [3, с. 23, 24] и с $\S 11)$. Тогда $x=\infty$ является корнем $g\left(d a^{1}, x\right)-g\left(d a^{0}, x\right)$, поскольку

$$
g\left(d a^{1}, \infty\right)-g\left(d a^{0}, \infty\right)=\int_{0}^{\infty} d a^{1}(s)-\sum_{j=1}^{m} a_{j}^{0}=0 .
$$


Так как $\Pi \leqslant 2 m\left(\Pi=2 m\right.$, если $g_{0}(x), \ldots, g_{m}(x)$ положительны и $a_{j}^{0}-a_{j}>0$ при всех $j, 1 \leqslant j \leqslant m$ ), то по теореме Гантмахера-Крейна число корней функции $g\left(d a^{1}, x\right)-g\left(d a^{0}, x\right), 0 \leqslant x \leqslant \infty$, не превосходит $2 m$, т.е. множество

$$
\left\{x \in[0,1] \mid \sum_{j=1}^{m} \frac{a_{j}^{0}}{s_{j}^{0}+x}=\int_{0}^{\infty} \frac{d a^{1}(s)}{s+x}\right\}
$$

содержит меньше, чем $2 m$ точек.

30. $\mathrm{B}^{\langle 32\rangle}$ рассказано, как доказать более обшую лемму.

31. Оценим $r_{1}(s)=d_{10}+d_{11} /\left(s+w_{11}\right)$ сверху при условии, что $0<w_{11}<1$. Так как $d_{10}<r_{1}\left(s_{1}\right)=\varphi\left(s_{1}\right)<1 / \sqrt{s_{1}}$ и $d_{11}<r(0)=\varphi(0)=2 /(\pi \sqrt{t})$, то

$$
r_{1}(s)=d_{10}+\frac{d_{11}}{s+w_{11}}<d_{10}+\frac{d_{11}}{s}<\frac{1}{\sqrt{s_{1}}}+\frac{2}{\pi s \sqrt{t}} .
$$

Теперь оценим $r_{1}(s)$ снизу. При $s>1$ и $0<t \leqslant 1$

$$
r_{1}(s) \geqslant \varphi(s)>\frac{2 \operatorname{arctg} 1}{\pi \sqrt{s}}=\frac{1}{2 \sqrt{s}}
$$

Полученное неравенство $1 /(2 \sqrt{s})<1 / \sqrt{s_{1}}+2 /(\pi s \sqrt{t})$ дает искомую оценку для $s_{1}$. Действительно, функция $1 /(2 \sqrt{s})-2 /(\pi s \sqrt{t})$ достигает своего максимума при $s \geqslant 1$ в точке

$$
s=s_{0}=\frac{(8 / \pi)^{2}}{t} \text {. }
$$

Поэтому

$$
\frac{1}{\sqrt{s_{1}}}>\left(\frac{1}{2 \sqrt{s_{0}}}-\frac{2}{\pi s_{0} \sqrt{t}}\right)=\frac{\pi \sqrt{t}}{32}, \text { где } s_{1}<\frac{c}{t},
$$

где $c=(32 / \pi)^{2}$, что и требовалось доказать.

32. Неравенство $w_{m 1}<w_{1}$ доказывается по той же схеме, что лемма 2 в $\langle 16\rangle$. Снова разобьем доказательство на три части: $\mathrm{A}^{\prime}$ ) совпадает с $\mathrm{A}$ ) в ${ }^{\langle 16\rangle}, \mathrm{B}^{\prime}$ ) и $\mathrm{C}^{\prime}$ ) являются модификациями В) и С) из ${ }^{\langle 16\rangle}$.

$\left.\mathrm{B}^{\prime}\right)$ Взяв малое $\varepsilon>0$, рассмотрим последовательность наборов

$$
\mathscr{I}_{n}=\left\{s_{n, 1}, \ldots, s_{n, m+1}\right\}, \quad s_{n, 1}>\cdots>s_{n, m+1}>0, \quad n=1, \ldots, N
$$

обладаюшую следующими тремя свойствами (ср. с $\langle 16\rangle)$ :

а) $\mathscr{S}_{1}=\mathscr{S}$, б) $s_{N, 2}<\varepsilon$, в) точки наборов $\mathscr{S}_{n}$ и $\mathscr{S}_{n+1}$ чередуются:

$$
s_{1}=s_{n, 1}=s_{n+1,1}>\cdots>s_{n, m+1}>s_{n+1, m+1}>0, \quad 1 \leqslant n<N .
$$

Согласно А) (см. $\left.{ }^{\langle 16\rangle}\right)$ для аппроксимаций $r\left(\mathscr{S}_{n}, s\right)$ и $r\left(\mathscr{S}_{n+1}, s\right)$

$$
w_{n, 1}>w_{n+1,1}>\cdots>w_{n, m+1}=w_{n+1, m+1}=0 .
$$

В частности,

$$
w_{1}=w_{1,1}>w_{N, 1}
$$


$\left.\mathrm{C}^{\prime}\right)$ При $\varepsilon \rightarrow 0$ аппроксимация $r\left(\mathscr{S}_{N}, s\right)$, очевидно, стремится к аппроксимации $r_{m}(s)$. Отсюда ввиду (120) следует искомое неравенство $w_{m 1}<w_{1}$.

33. Неравенство $w_{11}<w_{m 1}$ следует из цепочки неравенств

$$
w_{11}<w_{21}<\cdots<w_{m-1,1}<w_{m 1}
$$

которые доказываются так же, как в А) в $\langle 16\rangle$.

Например, неравенство $w_{m-1,1}<w_{m 1}$ проверяется следуюшим образом.

Разность

$$
r_{m}(s)-r_{m-1}(s)=d_{m, 0}-d_{m-1,0}+\sum_{k=1}^{m} \frac{d_{m, k}}{s+w_{m, k}}-\sum_{k=1}^{m-1} \frac{d_{m-1, k}}{s+w_{m-1, k}}
$$

запишем в виде

$$
c_{0}+\sum_{k=1}^{p} \frac{c_{k}}{s+x_{k}}, \quad x_{1}>\cdots>x_{p}>0, \quad p \leqslant 2 m-1 .
$$

Ввиду условий

$$
\begin{gathered}
r_{m}^{(k)}(0)=r_{m-1}^{(k)}(0), \quad 0 \leqslant k \leqslant 2 m-4, \\
r_{m}\left(s_{1}\right)=r_{m-1}\left(s_{1}\right), \quad r_{m}^{\prime}\left(s_{1}\right)=r_{m-1}^{\prime}\left(s_{1}\right)
\end{gathered}
$$

сумма $K$ кратностей корней рассматриваемой разности на полуоси $s \geqslant 0$ не меньше $2 m-1$. Значит, число П перемен знака в последовательности коэффициентов $\left\{c_{0}, \ldots, c_{p}\right\}$ не меньше $2 m-1$. Отсюда следует, что

$$
p=\Pi=2 m-1,
$$

а значит, полюсы $w_{m-1, k}$ и $w_{m, k}$ чередуются:

$$
w_{m, 1}>w_{m-1,1}>\cdots>w_{m, m-1}>w_{m-1, m-1}>w_{m, m},
$$

при любом другом их порядке $\Pi<2 m-1$.

34. В геофизике нередко рассматривают кусочно-постоянные скоростные функции $u(y)$. Нетрудно проверить, что условие (96) означает, что годограф Г соответствует именно такой скоростной функции (кусочно-постоянной на каждом конечном отрезке оси $y>0$ и принимаюшей в волноводе $\widehat{n}$ значений).

35. Докажем поочередно утверждения 6 и 7 , используя следующие обозначения. Пусть последовательность $t_{i} \in[0,1]$ сходится к $t^{\circ}$ при $i \rightarrow \infty$. Положим

$$
\begin{gathered}
n_{i}=n\left(t_{i}\right), \quad n^{\circ}=n\left(t^{\circ}\right) ; \\
a_{j}^{i}=a_{j}\left(t_{i}\right), s_{j}^{i}=s_{j}\left(t_{i}\right), \quad 1 \leqslant j \leqslant n_{i} ; \quad a_{j}^{\circ}=a_{j}\left(t^{\circ}\right), \quad s_{j}^{\circ}=s_{j}\left(t^{\circ}\right), \quad 1 \leqslant j \leqslant n^{\circ} ; \\
d a_{i}=d a\left(s, t_{i}\right)=\sum a_{j}^{i} \delta\left(s-s_{j}^{i}\right) d s, g_{i}(x)=g\left(d a_{i}, x\right)=\sum \frac{a_{j}^{i}}{s_{j}^{i}+x}, \quad 1 \leqslant j \leqslant n_{i} ; \\
d a^{\circ}=d a\left(s, t^{\circ}\right)=\sum a_{j}^{\circ} \delta\left(s-s_{j}^{\circ}\right) d s, g^{\circ}(x)=g\left(d a^{\circ}, x\right)=\sum \frac{a_{j}^{\circ}}{s_{j}^{\circ}+x}, \quad 1 \leqslant j \leqslant n^{\circ} .
\end{gathered}
$$

Коэффициенты $b_{k}\left(t_{i}\right)$ и полюсы $x_{k}\left(t_{i}\right)$ функций $r\left(s, t_{i}\right)$ (см. (100)) обозначим через $b_{k}^{i}, 0 \leqslant k \leqslant n_{i}$, и $x_{k}^{i}, 1 \leqslant k \leqslant n_{i}$, для коэффициентов $b_{k}\left(t^{\circ}\right)$ и полюсов $x_{k}\left(t^{\circ}\right)$ функции $r\left(s, t^{\circ}\right)$ введем обозначения $b_{k}^{\circ}, 0 \leqslant k \leqslant n^{\circ}$, и $x_{k}^{\circ}, 1 \leqslant k \leqslant n^{\circ}$, и, наконец, саму функцию $r\left(s, t^{\circ}\right)$ обозначим через $r^{\circ}(s)$. 
ДоКАЗАТЕЛЬСТВо УТВЕРЖДЕНИЯ 6. Разобьем доказательство на три части.

$\left.1^{\circ}\right)$ Проверим, что

А) для $x \in(0,1]$ при $i \rightarrow \infty$ функиии $g_{i}(x)$ сходятся $\kappa g^{\circ}(x)$ вместе со всеми производными ;

B) $n_{i} \geqslant n^{\circ}$, начиная с некоторого $i$.

Согласно (98) $n_{i} \leqslant \widehat{n}$. Числа $a_{j}^{i}$ ограничены: $0<a_{j}^{i} \leqslant 1$ и $\sum_{j=1}^{n_{i}} a_{j}^{i}=1$. При условии (96) согласно [2] числа $s_{j}^{i}$ тоже ограничены:

$$
0<s_{n_{i}}^{i}<\cdots<s_{1}^{i}<\widehat{s}_{1} .
$$

ЗАМЕчАниЕ. Если $t^{\circ}>0$, то $n_{i}, a_{j}^{i}$ и $s_{1}^{i}$ ограничены (по теоремам 1 и 6 ) и без предположения о том, что мажоранта удовлетворяет условию (96).

Ввиду ограниченности $n_{i}, a_{j}^{i}$ и $s_{j}^{i}$ будем считать (перейдя, если нужно, к подпоследовательности), что $n_{i}$ не зависят от $i: n_{i} \equiv n$, и сушествуют пределы

$$
s_{j}^{*}=\lim _{i \rightarrow \infty} s_{j}^{i}, \quad a_{j}^{*}=\lim _{i \rightarrow \infty} a_{j}^{i}, \quad 1 \leqslant j \leqslant n .
$$

Для этих пределов вследствие (121) при некотором $m \leqslant n$

$$
0<s_{m}^{*} \leqslant \cdots \leqslant s_{1}^{*}, \quad s_{j}^{*}=0 \text { при } j>m
$$

и (это доказывается так же, как (117), см. ${ }^{\langle 19\rangle}$ )

$$
a_{j}^{*} \geqslant 0 \text { при } 1 \leqslant j \leqslant m, a_{j}^{*}=0 \text { при } j>m, \text { причем } \sum_{j=1}^{m} a_{j}^{*}=1 .
$$

Положим

$$
d a^{*}=d a^{*}(s)=\sum_{j=1}^{m} a_{j}^{*} \delta\left(s-s_{j}^{*}\right) d s, \quad g^{*}(x)=g\left(d a^{*}, x\right), \quad \widehat{g}(x)=g(d \hat{a}, x) .
$$

При $i \rightarrow \infty$ функции $g_{i}(x)$ сходятся к $g^{*}(x)$ вместе со всеми производными и $g_{i}(x) \leqslant$ $\widehat{g}(x)$ на $(0,1]$. Поэтому и $g^{*}(x) \leqslant \widehat{g}(x)$ на $[0,1]$, т.е. (см. $\S 6$ от (46) до леммы 1$)$ $d a^{*} \prec d \hat{a}$. Так как $g_{i}(0)=\sum_{j=1}^{n_{i}} a_{j}^{i} / s_{j}^{i} \leqslant \widehat{g}(0)$, а значит, $a_{j}^{i} \leqslant \widehat{g}(0) s_{j}^{i}$, и так как $\kappa\left(s, t_{i}\right) \leqslant 1 / \sqrt{s}$, то $h\left(d a_{i}, t_{i}\right)=\sum_{j=1}^{n_{i}} a_{j}^{i} \kappa\left(s_{j}^{i}, t_{i}\right)$ сходятся при $i \rightarrow \infty \mathrm{K} h\left(d a^{*}, t^{\circ}\right)$. Виведем отсюда, что $d a^{*} \equiv d a^{\circ}$.

По определению мер $d a_{i}$ и $d a^{\circ}$

$$
\begin{aligned}
h\left(d a_{i}, t_{i}\right) & =\max h\left(d a, t_{i}\right) \text { по всем } d a \prec d \hat{a}, \\
h\left(d a^{\circ}, t^{\circ}\right) & =\max h\left(d a, t^{\circ}\right) \text { по всем } d a \prec d \hat{a} .
\end{aligned}
$$

Значит, для любого $\varepsilon>0$ найдется столь большое $i_{\circ}$, что при $i>i_{\circ}$ выполняется неравенство $h\left(d a_{i}, t_{i}\right) \geqslant h\left(d a^{\circ}, t_{i}\right)>h\left(d a^{\circ}, t^{\circ}\right)-\varepsilon$. Следовательно,

$$
h\left(d a^{*}, t^{\circ}\right)=\lim _{i \rightarrow \infty} h\left(d a_{i}, t_{i}\right) \geqslant h\left(d a^{\circ}, t^{\circ}\right),
$$

так что $h\left(d a^{*}, t^{\circ}\right)=\max h\left(d a, t^{\circ}\right)$ по всем $d a \prec d \hat{a}$ и (по утверждению 5$)$

$$
d a^{\circ} \equiv d a^{*} .
$$

Утверждения А) и В) следуют из (122); еше одно следствие:

$$
\left\{s_{1}^{\circ}, \ldots, s_{n^{\circ}}^{\circ}\right\} \text { - подпоследовательность }\left\{s_{1}^{*}, \ldots, s_{m}^{*}\right\} \text {. }
$$


ПояснЕниЕ. Вскоре мы докажем, что $n^{\circ}=m$ и последовательности $s_{j}^{\circ}$ и $s_{j}^{*}$ в (123) совпадают, но пока мы утверждаем лишш, что $n^{\circ} \leqslant m$ и что $s_{j}^{\circ}$ является подпоследовательностью $s_{j}^{*}$ (a priori могло бы оказаться, что при $1 \leqslant j \leqslant m$ и при $i \rightarrow \infty$ некоторые $s_{j}^{i}$ сливаются друг с другом или некоторые $\left.a_{j}^{i} \rightarrow 0\right)$.

$\left.2^{\circ}\right)$ Числа $x_{k}^{i}$ ограничены: $0 \leqslant x_{k}^{i} \leqslant 1$. Отсюда и из совпадения $r\left(s, t_{i}\right)$ и $\kappa\left(s, t_{i}\right)$ при $s=s_{1}^{i}$ следует, что числа $b_{k}^{i}$ тоже ограничены:

$$
b_{k}^{i}>0, \quad 0 \leqslant k \leqslant n, \quad \sum_{k=0}^{n} b_{k}^{i}<\left(s_{1}^{i}+1\right) \kappa\left(s_{1}^{i}, t_{i}\right) .
$$

Поэтому (снова перейдя к подпоследовательности) будем считать, что существуют пределы

$$
x_{k}^{*}=\lim _{i \rightarrow \infty} x_{k}^{i}, \quad 1 \leqslant k \leqslant n, \quad b_{k}^{*}=\lim _{i \rightarrow \infty} b_{k}^{i}, \quad 0 \leqslant k \leqslant n .
$$

Вследствие (100) при некотором $p, 1 \leqslant p \leqslant n$,

$$
0<x_{p}^{*} \leqslant \cdots \leqslant x_{1}^{*}=1, \quad x_{k}^{*}=0 \text { при } k>p .
$$

Докажем (ср. с (123)), что

$$
\left\{x_{1}^{*}, \ldots, x_{p}^{*}\right\}-\text { подпоследовательность }\left\{x_{1}^{\circ}, \ldots, x_{n^{\circ}}^{\circ}\right\} .
$$

Из утверждения 5 следует, что разность $\widehat{g}(x)-g^{\circ}(x)$ имеет нули кратности 1 или 2 в особых точках $x_{k}^{\circ}$ меры $d a^{\circ}$ и положительна в остальных точках $x \in[0,1]$. Разность $\widehat{g}(x)-g_{i}(x)$ неотрицательна на $[0,1]$ и равна нулю в особых точках $x_{k}^{i}$ меры $d a_{i}$. Поэтому (вследствие А) $) x_{k}^{*} \in\left\{x_{1}^{\circ}, \ldots, x_{n^{\circ}}^{\circ}\right\}, 1 \leqslant k \leqslant p$, и $x_{1}^{*}, \ldots, x_{p}^{*}$ все различны (иначе у $\widehat{g}(x)-g^{\circ}(x)$ имелся бы нуль кратности $K>2$ ), (124) доказано. Из (124) следует, что

$$
p \leqslant n^{\circ} \text { при } x_{n^{\circ}}^{\circ}>0, \quad p \leqslant n^{\circ}-1 \text { при } x_{n^{\circ}}^{\circ}=0 .
$$

$\left.3^{\circ}\right)$ Сравним

$$
r^{*}(s)=b_{0}^{*}+\sum_{k=1}^{n} \frac{b_{k}^{*}}{s+x_{k}^{*}} \quad \text { и } r^{\circ}(s)=b_{0}^{\circ}+\sum_{k=1}^{n^{\circ}} \frac{b_{k}^{\circ}}{s+x_{k}^{\circ}}
$$

с $\varphi(s)=\kappa\left(s, t^{\circ}\right)$. Из утверждения 5 следует, что разность $r^{\circ}(s)-\varphi(s)$ имеет двукратные нули в точках $s=s_{n^{\circ}}^{\circ}, \ldots, s_{1}^{\circ}$ и положительна в остальных точках $s>0$. Значит, сумма $K^{\circ}$ кратностей корней $r^{\circ}(s)-\varphi(s)$ равна $2 n^{\circ}$. Функции $r^{*}(s)$ и $\varphi(s)$, очевидно, совпадают в точках $s_{m}^{*}, \ldots, s_{1}^{*}$ вместе с производными, причем, если $q$ из этих точек сливаются: $s_{j+q-1}^{*}=\cdots=s_{j}^{*}$, то корень $s_{j}^{*}$ разности $r^{*}(s)-\varphi(s)$ имеет кратность $k_{j} \geqslant 2 q$. Поэтому сумма $K^{*}$ кратностей корней $r^{*}(s)-\varphi(s)$ не меньше $2 m$ и, учитывая (123),

$$
K^{*} \geqslant 2 m \geqslant 2 n^{\circ}=K^{\circ} .
$$

Докажем, что верны следуюшие утверждения:

C) $е с л и n^{\circ}>1$, mo $r^{*}(s) \equiv r^{\circ}(s)$;

D) $p=n^{\circ}$ при $x_{n^{\circ}}^{\circ}>0 ; p=n^{\circ}-1$ при $x_{n^{\circ}}^{\circ}=0 ;$ в последовательностях $(124)$ $x_{k}^{\circ}$ u $x_{k}^{*}$ совпадают при $1 \leqslant k \leqslant p$;

Е) $m=n^{\circ}$ и последовательности $s_{j}^{\circ} u s_{j}^{*}$ в (123) совпадают. 
ДоКАЗАТЕЛЬСТво. Пусть $r^{*}(s) \not \equiv r^{\circ}(s)$, тогда ввиду (123) разность

$$
\rho(s)=r^{*}(s)-r^{\circ}(s)=\left(r^{*}(s)-\varphi(s)\right)-\left(r^{\circ}(s)-\varphi(s)\right)
$$

имеет $n^{\circ}$ кратных корней $s_{1}^{\circ}, \ldots, s_{n}^{\circ}$ и, значит, сумма $K$ кратностей корней $\rho(s)$ не меньше $2 n^{\circ}$. Согласно (124)

$$
\begin{gathered}
\rho(s)=c_{0}+\sum_{k=1}^{n^{\circ}} \frac{c_{k}}{s+x_{k}^{\circ}} \text { при } x_{n^{\circ}}^{\circ}=0, \\
\rho(s)=c_{0}+\sum_{k=1}^{n^{\circ}} \frac{c_{k}}{s+x_{k}^{\circ}}+\frac{c}{s} \text { при } x_{n^{\circ}}^{\circ}>0, \text { где } c=\sum_{p<k \leqslant n} b_{k}^{*} \geqslant 0 .
\end{gathered}
$$

Число П перемен знака в последовательности коэффициентов $\rho(s)$ не больше $n^{\circ}$ в случае (127) и $\Pi \leqslant n^{\circ}+1$ в случае (128). По теореме Гантмахера-Крейна $K \leqslant \Pi$. Подставляя сюда найденные оценки для $K$ и П, получаем, что при $r^{*}(s) \not \equiv r^{\circ}(s)$

$$
2 n^{\circ} \leqslant K \leqslant \Pi \leqslant n^{\circ}+1 \text {, } \text { т.e. } n^{\circ}=1 \text {, }
$$

и утверждение C) доказано. А из него, из (124) и (125) легко следует и утверждение D).

Продолжая (129), получаем: если $r^{*}(s) \not \equiv r^{\circ}(s)$, то $\mathrm{K}=\Pi=2$, так что в (128) $c>0$ и $r^{*}(s)>r^{\circ}(s)$ при $s>0, s \neq s_{1}^{\circ}$. Таким образом,

$$
r^{*}(s) \geqslant r^{\circ}(s)>\varphi(s) \text { при } s>0, s \neq s_{1}^{\circ}, \ldots, s_{n^{\circ}}^{\circ}
$$

Поэтому $K^{*} \leqslant K^{\circ}$ и, с учетом (126), $m=n^{\circ}$, т.е. ввиду (123) утверждение Е) доказано.

А вместе с В), D) и Е) доказано и утверждение 6.

ДОКАЗАТЕЛЬСТВО УТВЕРЖДЕНИЯ 7. ВВиду (130) из СоОтношений

$$
r\left(s_{n}^{i}, t_{i}\right)=\kappa\left(s_{n}^{i}, t_{i}\right)<\kappa\left(0, t_{i}\right)
$$

при $i \rightarrow \infty$ следует: если $t^{\circ}>0$ - точка разрыва функции $n(t)$ (так что $\left.n \geqslant n^{\circ}+1 u s_{n}^{i} \rightarrow 0\right), m o$

$$
r^{\circ}(0)=\varphi(0) .
$$

Вьведем из (131) утверждение 7 . Допустим, что $x_{n}^{\circ} \leqslant t^{\circ}$ и, следовательно, $x_{k}^{\circ} \leqslant t^{\circ}<x_{k-1}^{\circ}$ для некоторого $k \leqslant n^{\circ}$. Положим $d \theta(x)=(2 / \pi) d \sqrt{x}$. Тогда

$$
\varphi(s)=\int_{t^{\circ}}^{\infty} \frac{d \theta(x)}{s+x}=\varphi_{1}(s)+\varphi_{2}(s)+\cdots+\varphi_{k}(s),
$$

где

$$
\varphi_{k}(s)=\int_{t^{\circ}}^{x_{k-1}^{\circ}} \frac{d \theta(x)}{s+x}, \ldots, \varphi_{2}(s)=\int_{x_{2}^{\circ}}^{x_{1}^{\circ}} \frac{d \theta(x)}{s+x}, \varphi_{1}(s)=\int_{x_{1}^{\circ}}^{\infty} \frac{d \theta(x)}{s+x} .
$$


По теореме Гантмахера-Крейна сумма $K^{\circ}$ кратностей корней разности $r^{\circ}(s)-\varphi(s)$ при $s \geqslant 0$ не больше числа $\Pi^{\circ}$ перемен знака в разложении

$b_{0}^{\circ}-\varphi_{1}(s)+\frac{b_{1}^{\circ}}{s+x_{1}^{\circ}}-\varphi_{2}(s)+\cdots+\frac{b_{k-1}^{\circ}}{s+x_{k-1}^{\circ}}-\varphi_{k}(s)+\left(\frac{b_{k}^{\circ}}{s+x_{k}^{\circ}}+\cdots+\frac{b_{n^{\circ}}^{\circ}}{s+x_{n^{\circ}}^{\circ}}\right)$.

Так как $\Pi^{\circ}=2 k \leqslant 2 n^{\circ}$ и так как ввиду (131) $K^{\circ} \geqslant 2 n^{\circ}+1$, то неравенство $K^{\circ} \leqslant \Pi^{\circ}$ нарушается. Значит, случай $x_{n^{\circ}}^{\circ} \leqslant t^{\circ}$ невозможен.

Итак, $0<t^{\circ}<x_{n^{\circ}}^{\circ}$. Возврашаясь к обозначениям из $\S 11$, получаем $(102)$.

36. По утверждению 6 нарушение монотонности $n(t)$ означает сушествование такой точки $t \in[0,1]$, что в любой ее окрестности есть точка $t^{*}>t$, в которой $n\left(t^{*}\right)>n(t)$.

37. По определению $P_{1}(x)=g^{*}(x)-g(x)$. По утверждению 5 , если $x_{j}^{*}$ не совпадает ни с одним $x_{k}, 1 \leqslant k \leqslant n(t)$, то

$$
g^{*}\left(x_{j}^{*}\right)=M\left(x_{j}^{*}\right)>g\left(x_{j}^{*}\right), \text { так что } P_{1}\left(x_{j}^{*}\right)>0 .
$$

В частности, числа $P_{1}\left(X_{Q}\right), \ldots, P_{1}\left(X_{q+1}\right)$ положительны.

38. Неравенство $\Pi \leqslant \Pi_{2}$ для числа перемен знака в последовательностях $\left\{A_{N}, \ldots, A_{1}\right\}$ и $\left\{P_{2}\left(S_{N}\right), \ldots, P_{2}\left(S_{1}\right)\right\}$ вытекает из следующих двух фактов.

$\left.1^{\circ}\right)$ Если $S_{i}$ не принадлежит пересечению множеств

$$
\left\{s_{j}\right\}, \quad 1 \leqslant j \leqslant n(t), \quad\left\{s_{j}^{*}\right\}, \quad 1 \leqslant j \leqslant n\left(t^{*}\right),
$$

то знаки $A_{i}$ и $P_{2}\left(S_{i}\right)$ противоположны. Чтобы доказать это, достаточно вспомнить определение $P_{2}(s)$ :

$$
P_{2}(s)=\left(r\left(s, t^{*}\right)-\kappa\left(s, t^{*}\right)\right)-(r(s, t)-\kappa(s, t)),
$$

и заметить, что (вследствие утверждения 5) знаки $a_{j}^{*} u-a_{j}$ противоположны знакам $P_{2}\left(s_{j}^{*}\right)$ и $P_{2}\left(s_{j}\right)$ :

$a_{j}^{*}>0, \quad P_{2}\left(s_{j}^{*}\right)<0, \quad 1 \leqslant j \leqslant n\left(t^{*}\right) ; \quad-a_{j}<0, \quad P_{2}\left(s_{j}\right)>0, \quad 1 \leqslant j \leqslant n(t)$.

$\left.2^{\circ}\right)$ Если же $S_{i} \in\left\{s_{j}\right\}_{j=1}^{n(t)} \cap\left\{s_{j}^{*}\right\}_{j=1}^{n\left(t^{*}\right)}$, то $P_{2}\left(S_{i}\right)=0$.

Отсюда (по правилу замены нулей при подсчете $\left.\Pi_{2}\right) \Pi \leqslant \Pi_{2}$.

39. Докажем, что знаки $B_{i}$ и $P_{1}\left(X_{i}\right)$ совпадают, если $X_{i}$ не принадлежит пересечению множеств

$$
\left\{x_{k}\right\}, \quad 1 \leqslant k \leqslant n(t), \quad\left\{x_{k}^{*}\right\}, \quad 1 \leqslant k \leqslant n\left(t^{*}\right) .
$$

Согласно ${ }^{\langle 37\rangle}$, если $x_{j}^{*}$ не совпадает ни с одним $x_{k}, 1 \leqslant k \leqslant n(t)$, то $P_{1}\left(x_{j}^{*}\right)>0$. Аналогично, если $x_{j}$ не совпадает ни с одним $x_{k}^{*}, 1 \leqslant k \leqslant n^{*}(t)$, то

$$
g\left(x_{j}\right)=M\left(x_{j}\right)>g^{*}\left(x_{j}\right), \text { так что } P_{1}\left(x_{j}\right)<0 .
$$


Итак, при указанных условиях

$$
b_{j}^{*}>0, \quad P_{1}\left(x_{j}^{*}\right)>0, \quad-b_{j}<0, \quad P_{1}\left(x_{j}\right)<0 .
$$

Поскольку, кроме того,

$$
P_{1}\left(X_{i}\right)=0, \text { если } X_{i} \in\left\{x_{k}\right\}_{k=1}^{n(t)} \cap\left\{x_{k}^{*}\right\}_{k=1}^{n\left(t^{*}\right)},
$$

то (по правилу замены нулей при подсчете $\left.\Pi_{1}^{\prime}\right) \Pi^{\prime} \leqslant \Pi_{1}^{\prime}$.

40. Так как $P_{2}(s)=\left(r\left(s, t^{*}\right)-\kappa\left(s, t^{*}\right)\right)-(r(s, t)-\kappa(s, t))$ и $r\left(s, t^{*}\right) \geqslant \kappa\left(s, t^{*}\right)$, a $r(s, t) \geqslant \kappa(s, t)$ при $s \geqslant 0$, то точки $S_{j}$ в последовательности

$$
\left\{P_{2}\left(S_{N}\right), \ldots, P_{2}\left(S_{1}\right)\right\}
$$

обладают важным свойством: в них либо $r(s, t)-\kappa(s, t)$, либо $r\left(s, t^{*}\right)-\kappa\left(s, t^{*}\right)$, либо обе эти разности обращаются в нуль вместе с производной по $s$.

Последнее выполняется, если $S_{j}$ - корень $P_{2}(s)$. Поэтому, если $P_{2}\left(S_{j}\right)=0$, то $S_{j}-$ кратныи корень $P_{2}(s)$.

41. Положим (см. (96)) $\hat{g}(x)=g(d \hat{a}, x)$ и по аналогии с

$$
P_{2}(s)=\left(r\left(s, t^{*}\right)-\kappa\left(s, t^{*}\right)\right)-(r(s, t)-\kappa(s, t))
$$

представим $P_{1}(x)=g^{*}(x)-g(x)$ в виде

$$
P_{1}(x)=(\widehat{g}(x)-g(x))-\left(\widehat{g}(x)-g^{*}(x)\right) .
$$

Так как $\widehat{g}(x) \geqslant g(x), \widehat{g}(x) \geqslant g^{*}(x)$ при $x \in[0,1]$, то $X_{k}, 1<k<Q$, принадлежашие интервалу $(0,1)$, играют в последовательности

$$
\left\{P_{1}\left(X_{Q}\right), \ldots, P_{1}\left(X_{k}\right), \ldots, P_{1}\left(X_{1}\right), P_{1}\left(X_{0}\right)\right\}
$$

особую роль: в них $\widehat{g}(x)$ совпадает вместе с производной либо с $g(x)$, либо с $g^{*}(x)$, либо с обеими этими функциями.

Последнее выполняется, если $X_{k}, 1<k<Q,-$ корень $P_{1}(x)$. Поэтому, если $P_{1}\left(X_{k}\right)=0,1<k<Q$, то $X_{k}-$ кратный корень $P_{1}(x)$.

То же верно для $X_{Q}$, если $X_{Q}>0$, и для $X_{1}$, если $X_{1}<1$.

При $X_{Q}=0$ и $X_{1}=1$ корни $X_{Q}$ и $X_{1}$, как правило, простые; поэтому обычно обрашение в нуль $P_{1}\left(X_{Q}\right)$ и $P_{1}\left(X_{1}\right)$ добавляет к $K_{1}$ (каждое) лишь по единице; корню $X_{0}=+\infty$ мы условились приписывать кратность 1 ; поэтому обрашение в нуль $P_{1}\left(X_{0}\right)$ тоже добавляет к $K_{1}$ лишь единицу. Но и к $\Pi_{1}$ (при вычислении $\Pi_{1}$ по правилу замены нулей) обрашение в нуль $P_{1}\left(X_{Q}\right), P_{1}\left(X_{1}\right)$ и $P_{1}\left(X_{0}\right)$ тоже добавляет лишь по единице. Поэтому $\Pi_{1} \leqslant K_{1}$.

42. Модифицируя приведенное доказательство теоремы 9 , можно показать, что функция $n(t)$ непрерьвна при $t=0$ и, следовательно, не убывает не только на полуинтервале $(0,1]$, но и на отрезке $[0,1]$.

43. Для различения основного и исключительного случаев используем классификацию мер из [2], приведенную в $\S 6:$ при любом $t \in[0,1]$ любая дискретная мера $d a$ принадлежит либо $\mathscr{G}(t)$, либо $\mathscr{M}(t)=\mathscr{E}(t) \cup \mathscr{L}(t)$. Согласно [2] (это доказывается так же, как (69)), если dâ $\in \mathscr{M}(t)$, mo $h(d \hat{a}, t)=\max h(d a, t)$ по всем $d a \prec d \hat{a}$. 
ЛЕмма 11. Для любих $t u t^{0}, 0 \leqslant t^{0}<t \leqslant 1$, справедливо включение $\mathscr{G}(t) \supseteq \mathscr{G}\left(t^{0}\right)$.

СлЕДСтВИЕ. Возъмем разбиение дискретных мер на $\mathscr{G}$ u $\mathscr{M}$ при $t=0$, тогда по лемме 11 основной и исключительный случаи характеризуются, соответственно, условиями dâ $\in \mathscr{G}$ и dâ $\in \mathscr{M}=\mathscr{E} \cup \mathscr{L}$.

ДокАЗАтЕЛЬСтво лЕммы 11. Лемма вытекает из следуюшего легко проверяемого $^{\langle 44\rangle}$ утверждения.

Положим $\varphi^{0}(s)=\kappa\left(s, t^{0}\right), \varphi(s)=\kappa(s, t), 0 \leqslant t^{0}<t \leqslant 1$. Пусть $r^{0}(s)$ u $r(s)$ - рачиональные аппроксимации (41), (42) функций $\varphi^{0}(s)$ и $\varphi(s)$ для одного и того же набора точек $\mathscr{S}$ в (40), и пусть точка $t$ достаточно близка $\kappa t^{0}$. Тогда полюсъ $w_{j}$ и $w_{j}^{0}$ аппроксимаций $r(s)$ и $r^{0}(s)$ чередуются:

$$
w_{1}>w_{1}^{0}>\cdots>w_{m}>w_{m}^{0}>w_{m+1}=w_{m+1}^{0}=0 .
$$

ДоказАтельство теоремы 10 . Так как $t_{1}=\frac{3}{5}-$ первый член универсальной последовательности $\left\{t_{k}\right\}$ (квадрат положительного корня полинома Лежандра $\left.L_{3}(x)=\frac{5}{2} x^{3}-\frac{3}{2} x\right)$, то по утверждению 1 из $\S 5$ при $t \geqslant \frac{3}{5}$ и в основном, и в исключительном случае $n(t) \equiv 1$.

Следовательно, при $\widehat{n}>1$ для некоторого $\widehat{t} \in\left[0, \frac{3}{5}\right]$ максимум $h(d a, \widehat{t})$ достигается не на мере $d \hat{a}$, т.е. $d \hat{a} \in \mathscr{G}(\widehat{t})$. Положим $t^{0}=\inf \{\hat{t}, d \hat{a} \in \mathscr{G}(\widehat{t})\}$. Ясно, что тогда в (132) $w_{1}^{0}=1$ и, значит, $w_{1}>1$, т.е. $d \hat{a} \in \mathscr{M}\left(t^{0}\right)$ и $d \hat{a} \in \mathscr{G}(t)$ npu всеx $t>t^{0}$.

44. Чтобы закончить доказательство леммы 11 и теоремы 10, остается проверить (132). Сначала докажем, что

$$
w_{m+1}^{0}=0 \leqslant t^{0}<w_{m}^{0}
$$

Пусть (133) выполняется, тогда при $d \theta(x)=(2 / \pi) d \sqrt{x}$

$$
\varphi^{0}(s)=\int_{t^{0}}^{\infty} \frac{d \theta(x)}{s+x}=\varphi_{0}^{0}(s)+\varphi_{1}^{0}(s)+\cdots+\varphi_{m}^{0}(s)
$$

где

$$
\varphi_{m}^{0}(s)=\int_{t^{0}}^{w_{m}^{0}} \frac{d \theta(x)}{s+x}, \quad \ldots, \quad \varphi_{1}^{0}(s)=\int_{w_{2}^{0}}^{w_{1}^{0}} \frac{d \theta(x)}{s+x}, \quad \varphi_{0}^{0}(s)=\int_{w_{1}^{0}}^{\infty} \frac{d \theta(x)}{s+x}
$$

По теореме Гантмахера-Крейна сумма $K^{0}$ кратностей корней $r^{0}(s)-\varphi^{0}(s)$ не больше числа $\Pi^{0}$ перемен знака в разложении

$$
r^{0}(s)-\varphi^{0}(s)=d_{0}^{0}-\varphi_{0}(s)+\frac{d_{1}^{0}}{s+w_{1}^{0}}-\varphi_{1}(s)+\cdots+\frac{d_{m}^{0}}{s+w_{m}^{0}}-\varphi_{m}(s)+\frac{d_{m+1}^{0}}{s} .
$$

Так как $s_{1}^{0}>\cdots>s_{m}^{0}>s_{m+1}^{0}-$ кратные корни $r^{0}(s)-\varphi^{0}(s)$, то в рассматриваемом случае неравенство $K^{0} \leqslant \Pi^{0}$ обрашается в равенство $K^{0}=\Pi^{0}=2 m+2$, а при нарушении (133) $\Pi^{0}$ становится менњше $K^{0}$, т.е. случай $t^{0} \geqslant w_{m}^{0}$ невозможен. 
Теперь применим теорему Гантмахера-Крейна к разности

$$
\Delta(s)=(r(s)-\varphi(s))-\left(r^{0}(s)-\varphi^{0}(s)\right)=r(s)-r^{0}(s)+\left(t-t^{0}\right) I(s),
$$

где

$$
I(s)=\frac{1}{t-t^{0}} \int_{t^{0}}^{t} \frac{d \theta(x)}{s+x},
$$

a $t$ столь близко к $t^{0}$, что $0 \leqslant t^{0}<t<w_{m}^{0}$.

Приведем в $\Delta(s)$ подобные члены и положим

$$
\Delta_{0}=d_{0}-d_{0}^{0}, \quad \Delta_{m+1}=d_{m+1}-d_{m+1}^{0}, \quad \Delta t=t-t^{0} .
$$

Сумма $K$ кратностей корней $\Delta(s)$ не больше числа П перемен знака в последовательности коэффициентов $\Delta(s)$. Так как $\Delta(s)$ имеет $m+1$ кратных корней $s_{1}^{0}>\cdots>s_{m}^{0}>s_{m+1}^{0}$, то $K \geqslant 2 m+2$. При вьполнении (132) П равно числу перемен знака в разложении

$$
\Delta(s)=\Delta_{0}+\frac{d_{1}}{s+w_{1}}-\frac{d_{1}^{0}}{s+w_{1}^{0}}+\cdots+\frac{d_{m}}{s+w_{m}}-\frac{d_{m}^{0}}{s+w_{m}^{0}}+\Delta t I(s)+\frac{\Delta_{m+1}}{s},
$$

т.е. (если $\Delta_{0}$ и $\left.\Delta_{m+1}<0\right) \Pi=2 m+2=K$, а при нарушении (132) $\Pi<K$.

Доказательство леммы 11 и теоремы 10 закончено.

\section{Список литературы}

1. Гервер М. Л. Рациональные аппроксимации, устойчивые многочлены и расслоения в задаче поиска самого шшрокого волновода // Геодинамика и прогноз землетрясений (Вычислительная сейсмология: Вьп. 26). М.: Наука, 1994. С. 176-201.

2. Гервер М. Л. Существование и единственность максимума и теоремы о конусах, 1-аппроксимациях и расслоениях для одного класса экстремальных задач // Препринт: МИТП РАН, 1994.

3. Гервер М.Л., Кудрявцева Е. А. Теорема об отношениях предшествования, генерируемых вполне положительными ядрами // Матем. сб. 1995. Т. 186. №9. С. 19-44.

4. Гервер M. Л., Кудрявиева E. A. О границе множества решений в задаче обрашения годографа // Докл. АН. 1996. Т. 346. № 5. С. 672-674.

5. Гервер M. Л., Кудряв цева E. А. Об экстремальных свойствах волноводов с конечным числом слоев в задаче обращения годограффа // Докл. АН. (в печати).

6. Gerver M. L., Kudryavtseva E. A. On the Boundary of the Set of Solutions in Travel Time Inversion and on Extremal Properties of Waveguides With a Finite Number of Layers // IUGG XXI General Assembly. Abstracts. Week B. Boulder, Colorado. July 2-14. 1995. P. B10 (UB31B-7).

7. Kudryavtseva E. A., Gerver M. L. An Algorithm Yielding the Universal Sequence $U$ in Travel Time Inversion // IUGG XXI General Assembly. Abstracts. Week B. Boulder, Colorado. July 2-14. 1995. P. B11 (UB31B-13).

8. Крейн М. Г., Нудельман А. А. Проблема моментов Маркова и экстремалњные задачи. М.: Наука, 1973.

9. Herglotz G. Über das Benndorfsche Problem der Fortpflanzungsgeschwindigkeit der Erdbebenstrahlen // Phys. Zeitschrift. Jahrgang 8. 1907. № 5. P. 145-147.

10. Bateman $H$. The solution of the integral equation which connects the velocity of propagation of an earthquake wave in the interior of the Earth with the times which the disturbance takes to travel to different stations on the Earth's surface // Phil. Mag. Ser. 6. 1910. V. 19. P. 576-587. 
11. Slichter $L$. B. The theory of interpretation of seismic travel-time curves in horizontal structures // Physics. 1932. V. 3. №6. P. 273-295.

12. Гервер М. Л., Маркушевич В. М. Исследование неоднозначности при определении по годографу скорости распространения сейсмической волны // Докл. АН СССР. 1965. T. 163. №6. C. 1377-1380.

13. Gerver M. L., Markushevich V. M. Determination of seismic wave velocity from the traveltime curve // Geophys. J. Royal Astr. Soc. 1966. V. 11. P. 165-173.

14. Гервер М. Л., Маркушевич В. М. Определение по годографу скорости распространения сейсмической волны // Методы и программы для анализа сейсмических наблюдений (Вычислительная сейсмология: Вьп. 3). М.: Наука, 1967. С. 3-51.

15. Кронрод А. С. Узлы и веса квадратурных формул. М.: Наука, 1964.

16. Полиа Г., Сеге Г. Задачи и теоремы из анализа. Ч. 2. М.: Наука, 1978.

17. Гервер М. Л. Несколько простых теорем о расположении корней // УМН. 1996. Т. 51. № 3. C. 191-192.

18. Карлин $C$., Стадден $B$. Чебышёвские системы и их применение в анализе и статистике. М.: Наука, 1976.

19. Гантмахер Ф.Р., Крейн М.Г. Осцилляционные матрицы и ядра и малые колебания механических систем. М.-Л.: ГИТТЛ, 1950.

20. Колмогоров A.H., Фомин C. В. Элементы теории функций и функционального анализа. М.: Наука, 1972.

Международный институт теории прогноза землетрясений и математической геофизики РАН;

Московский государственный

университет им. М.В. Ломоносова

E-mail: gerver@mitp.rssi.ru, m.1@gerver.mccme.ru
Поступила в редакцию 20.08.1996 